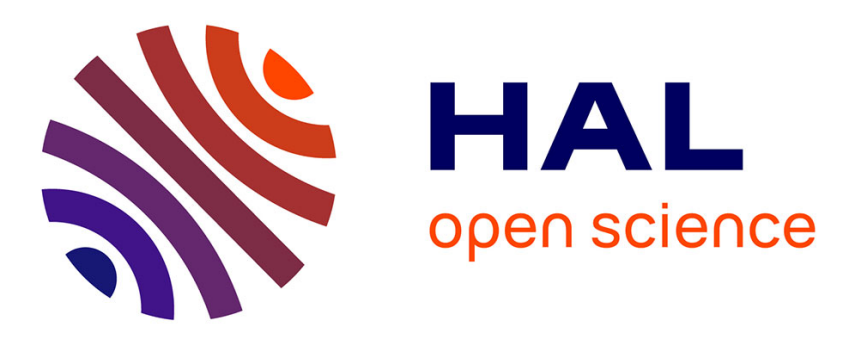

\title{
Generating a condensed representation for association rules
}

Nicolas Pasquier, Rafik Taouil, Yves Bastide, Gerd Stumme, Lotfi Lakhal

\section{To cite this version:}

Nicolas Pasquier, Rafik Taouil, Yves Bastide, Gerd Stumme, Lotfi Lakhal. Generating a condensed representation for association rules. Journal of Intelligent Information Systems, 2005, 24 (1), pp.29-60. 10.1007/s10844-005-0266-z . hal-00363015

\section{HAL Id: hal-00363015 https://hal.science/hal-00363015}

Submitted on 26 Apr 2010

HAL is a multi-disciplinary open access archive for the deposit and dissemination of scientific research documents, whether they are published or not. The documents may come from teaching and research institutions in France or abroad, or from public or private research centers.
L'archive ouverte pluridisciplinaire HAL, est destinée au dépôt et à la diffusion de documents scientifiques de niveau recherche, publiés ou non, émanant des établissements d'enseignement et de recherche français ou étrangers, des laboratoires publics ou privés. 


\title{
Generating a Condensed Representation for Association Rules
}

\author{
Nicolas Pasquier (nicolas.pasquier@unice.fr) \\ I3S (CNRS UMR 6070) - Université de Nice-Sophia Antipolis, 06903 Sophia Antipolis, France \\ Rafik Taouil (taouil@univ-tours.fr) \\ LI - Université Francois Rabelais de Tours, 3 place Jean Jaurès, 41000 Blois, France \\ Yves Bastide (yves.bastide@irisa.fr) \\ IRISA - INRIA Rennes, campus universitaire de Beaulieu, 35042 Rennes, France \\ Gerd Stumme (stumme@uni-kassel.de) \\ Fachbereich Mathematik/Informatik, Universität Kassel, 34121 Kassel, Germany \\ Lotfi Lakhal (lotfi.lakhal@lim.univ-mrs.fr) \\ LIM (CNRS FRE 2246) - Université de la Méditerranée, case 901, 13288 Marseille, France
}

\begin{abstract}
Association rule extraction from operational datasets often produces several tens of thousands, and even millions, of association rules. Moreover, many of these rules are redundant and thus useless. Using a semantic based on the closure of the Galois connection, we define a condensed representation for association rules. This representation is characterized by frequent closed itemsets and their generators. It contains the non-redundant association rules having minimal antecedent and maximal consequent, called min-max association rules. We think that these rules are the most relevant since they are the most general non-redundant association rules. Furthermore, this representation is a basis, i.e., a generating set for all association rules, their supports and their confidences, and all of them can be retrieved needless accessing the data. We introduce algorithms for extracting this basis and for reconstructing all association rules. Results of experiments carried out on real datasets show the usefulness of this approach. In order to generate this basis when an algorithm for extracting frequent itemsets - such as APRIORI for instance - is used, we also present an algorithm for deriving frequent closed itemsets and their generators from frequent itemsets without using the dataset.
\end{abstract}

Keywords: Data mining, Galois closure operator, frequent closed itemsets, generators, min-max association rules, basis for association rules, condensed representation.

\section{Introduction}

The purpose of association rule extraction, introduced in (Agrawal et al., 1993), is to discover significant relations between binary attributes, called items, in large datasets. An example of association rule extracted from a dataset of supermarket sales is: 'cereals $\wedge$ sugar $\rightarrow$ milk ( support $=7 \%$, confidence $=67 \%$ )'. This rule states that customers who buy cereals and sugar also tend to buy milk. The support measure defines the range of the rule, i.e., the proportion of customers who bought the three items among all customers. The confidence measure defines the precision of the rule, i.e., the proportion of customers who bought milk among those who bought cereals and sugar. Only rules with support and confidence above some minimal support and confidence thresholds, defined by the analyst according to the application, are extracted.

Classical approaches for mining association rules operate in two phases:

(C) 2008 Kluwer Academic Publishers. Printed in the Netherlands. 
1. Extracting frequent itemsets and their support from the dataset. Frequent itemsets are sets of items contained in a proportion of objects above the minimum support threshold.

2. Generating association rules from frequent itemsets and supports. Only rules with confidence above the minimum confidence threshold are generated.

The first phase is the most computationally intensive, since the number of potential frequent itemsets is exponential in the size of the set of items and several dataset scans, very expensive in execution times, are required to count their supports. Classical approaches can be classified into three main trends. Approaches in the first trend are based on the levelwise extraction of frequent itemsets (Agrawal and Srikant, 1994; Mannila et al., 1994). That is a breadth-first exploration of the search space where all potential frequent itemsets of a given size are considered simultaneously (Mannila and Toivonen, 1997). These approaches are efficient for mining association rules from weakly correlated data, such as market basket data, but performances drastically decrease when data are dense or correlated, such as statistical data for instance. Approaches in the second trend are based on the extraction of maximal ${ }^{1}$ frequent itemsets (Bayardo, 1998; Lin and Kedem, 1998; Zaki et al., 1997) to improve the efficiency. Once all maximal frequent itemsets are extracted, all frequent itemsets are derived and their support are counted in the dataset. In the third trend, approaches are based on the extraction of frequent closed itemsets (Pasquier et al., 1998; Zaki and Ogihara, 1998) defined using the Galois closure operator. These approaches first extract all frequent closed itemsets and then, both frequent itemsets and their support are derived from them, without dataset access. In the case of dense or correlated data, there are much fewer frequent closed itemsets than frequent itemsets and thus, these approaches improve the extraction efficiency compared to approaches in the first trend. Compared to approaches in the second trend, approches based on frequent closed itemsets can be more efficient in the case of correlated data due to the cost of generating all subsets of the maximal frequent itemsets and counting their support in the dataset.

Another major research topic in data mining is the problem of relevance and usefulness of extracted association rules. This problem is related to the number of extracted rules - that is most often very large - and to the important proportion of redundant rules, i.e. rules bringing the same information, among them. This problem becomes crucial when data are dense or correlated, such as statistical data, telecommunication data or nominative market basket data (Bayardo and al., 2000; Brin and al., 1997; Siverstein et al., 1998). For instance, using a census dataset sample constituted of 10,000 objects, each one containing values of 73 binary attributes, more than $2,000,000$ association rules with support and confidence above $90 \%$ were extracted. The analyst is then confronted with the following problems: How to handle such a list of association rules? Is it possible to reduce its size without losing information? Moreover, the inspection of extracted association rules shown that redundant rules represent the majority of them. Their suppression will thus considerably reduce the number of rules to be handled by the analyst. In the previous example, this

\footnotetext{
1 All maximal and minimal sets considered are defined according to the inclusion relation.
} 
suppression reduced the number of rules to a few thousands. In addition, redundant rules can be misleading as discussed in example 1 . Thus, the following question arises: How to reduce extracted association rules to a smaller list containing only non-redundant association rules?

Example 1. To illustrate the problem of redundant association rules, we present nine rules extracted from the Mushrooms dataset describing characteristics of 8416 mushrooms (Blake and Merz, 1998) in table I. These rules have identical supports and confidences, of $51 \%$ and $54 \%$ respectively, and the item "free gills" in the antecedent.

Table I. Redundant association rules.

\begin{tabular}{|l|ll|}
\hline 1) free gills $\rightarrow$ edible & 6) free gills, partial veil $\rightarrow$ edible, white veil \\
2) free gills $\rightarrow$ edible, partial veil & 7) free gills, white veil $\rightarrow$ edible \\
3) free gills $\rightarrow$ edible, white veil & 8) free gills, white veil $\rightarrow$ edible, partial veil \\
4) free gills $\rightarrow$ edible, partial veil, white veil & 9) free gills, partial veil, white veil $\rightarrow$ edible \\
5) free gills, partial veil $\rightarrow$ edible & \\
\hline
\end{tabular}

Obviously, rules 1 to 3 and 5 to 9 do not add any information to rule 4 since all these rules have identical supports and confidences. We thus say that these rules are redundant compared to rule 4, the most relevant from the analyst's point of view for it summarizes the nine rules. This rule has a minimal antecedent (left-hand side) and a maximal consequent (right-hand side) among the nine rules. Moreover, examining only one of these eight rules, say for instance rule 9, the analyst will believe that a mushroom has $54 \%$ chances to be edible if it has free gills and a partial white veil. As a matter of fact, it has $54 \%$ chances to be edible and have a partial white veil if it has free gills. Redundant rules can therefore be misleading and cause misinterpretations of the results. We believe that extracting only rule 4 will improve the result relevance.

In the rest of the paper, we differentiate exact association rules, noted $l \Rightarrow l^{\prime}$, that have a $100 \%$ confidence, and approximate association rules, noted $l \rightarrow l^{\prime}$, that have a confidence lower than $100 \%$. Exact association rules are valid for all objects in the dataset whereas approximate association rules are valid for a proportion of objects equal to their confidence.

\subsection{Related Work}

Approaches addressing this issue can be classified into three main trends. Approaches in the first trend provide mechanisms for filtering extracted association rules. In the two other trends, approaches "extend" the definition of association rules in order not to extract "similar" ones.

Approaches in the first trend allow the analyst to define some templates (Baralis and Psaila, 1997; Klemettinen and al., 1994), boolean operators (Bayardo and al., 2000; Ng et al., 1998; Srikant et al., 1997) or SQL-like operators (Meo et al., 1998) in order to select rules according to his/her preferences. In (Bayardo and al., 2000), 
boolean operators are coupled with further measures of "usefulness" of the rules. By selecting a subset of all extracted association rules, these approaches reduce the number of rules to handle during the visualization, but redundancies are not suppressed.

In the second trend, some approaches use a taxonomy of items to extract generalized association rules (Han and Fu, 1999; Srikant and Agrawal, 1995), i.e., association rules between sets of items that belong to different levels of the taxonomy. Some approaches use statistical measures, such as Pearson's correlation or $\chi^{2}$ test for instance, instead of the confidence to determine the precision of the rule (Brin and al., 1997; Morimoto et al., 1998; Siverstein et al., 1998). Other approaches in this trend allow to extract only rules with maximal antecedents among those with the same supports and the same consequents (Srikant and Agrawal, 1996; Toivonen et al., 1995). That is, a rule $r$ will be pruned if another rule $r^{\prime}$ has the same consequent and an antecedent that is a superset of the one of $r$. In example 1, rules 4,6 , 8 and 9 have maximal antecedents and will be extracted. Finally, the approach proposed in (Bayardo and Agrawal, 1999) identifies optimal rules according to several interestingness metrics (confidence, conviction, lift, Laplace, gain, etc.) and a partial order on the rules.

Approaches in the third trend make use of the closure of the Galois connection to extract bases, or reduced covers, for association rules. Informally, a basis is a non-redundant set that is minimal according to some mathematical property and from which all association rules are deducible, with support and confidence, without accessing the dataset. These bases are adaptations of the Duquenne-Guigues basis for global implications (Duquenne and Guigues, 1986; Ganter and Wille, 1999) and the Luxenburger basis for partial implications (Luxenburger, 1991). They were introduced in Formal Concept Analysis and their adaptation to the association rule framework is studied in (Pasquier et al., 1999c; Taouil et al., 2000; Zaki, 2000). In the Duquenne-Guigues basis for exact association rules, antecedents of rules are frequent pseudo-closed itemsets and consequents are frequent closed itemsets. In the Luxenburger basis for approximate association rules, both antecedents and consequents are frequent closed itemsets: We select approximate rules with both a maximal antecedent and a maximal consequent among rules having identical supports and confidences. In example 1, rule 9 will be the only one extracted. The union of the Duquenne-Guigues and the Luxenburger bases is a basis for all association rules. This basis is minimal with respect to the number of rules and, since for most data types there are much fewer frequent closed and pseudo-closed itemsets than there are frequent itemsets, it is very small. However, it does not contain non-redundant rules with minimal antecedent and maximal consequent.

In previous works about the pruning of redundant implication rules (functional dependencies), such as the canonical and the minimum covers definitions (Beeri and Bernstein, 1979; Maier, 1980), redundant rules are defined according to an inference system based on Armstrong axioms (Armstrong, 1974). However, these results cannot be directly applied to the association rule framework since redundant association rules cannot be defined according to this system: Supports and confidences are important information that must be considered to characterize redundant rules. Such an inference system for association rules does not exist to our knowledge. 
The idea behind non-redundant association rules as defined hereafter is to identify the most relevant rules, each one bringing the same information as several others.

\subsection{Contribution}

Our goal is to improve association rules relevance and usefulness by extracting as few rules as possible without losing information. To achieve this, we propose to generate a condensed representation (Mannila and Toivonen, 1996) by maximizing the information brought by each rule. As pointed out in example 1, we believe that the most relevant association rules are the most general ${ }^{2}$ non-redundant rules: Those with minimal antecedent and maximal consequent. Extracting such rules will improve the result usefulness, while reducing its size. Therefore, in the following:

- We define non-redundant association rules with minimal antecedent and maximal consequent, called min-max association rules. These rules are defined using the semantic for association rule extraction based on the Galois closure. Their antecedents and consequents are characterized by frequent closed itemsets and their generators (Pasquier et al., 1998).

- We show that the min-max association rules constitute a basis, called min-max basis for association rules. All association rules can be deduced by generating all the sub-rules of the min-max association rules, considering their supports and confidences.

- We propose efficient algorithms to generate the min-max basis from frequent closed itemsets and their generators, such as extracted by the Close (Pasquier et al., 1998; Pasquier et al., 1999b) and the A-Close (Pasquier et al., 1999a) algorithms. We also introduce algorithms to reconstruct all association rules, or a part of them, from this basis without having to access the data.

- We present the Close ${ }^{+}$algorithm that identifies frequent closed itemsets, their generators and their supports among frequent itemsets and their supports. This algorithm is simple and efficient since it does not require any dataset access. It enables the generation of the min-max basis when an algorithm for extracting all frequent itemsets, such as APRIORI (Agrawal and Srikant, 1994) for instance, is used.

Extracting min-max association rules minimizes as much as possible the number of rules while keeping the same information in the result: Only the most general non-overlapping association rules are extracted and therefore redundant rules are pruned. Since for many real datasets redundant rules represent the majority of extracted rules, the reduction will be almost always significant. This reduction will be considerable in the case of dense or correlated data for which the total number of rules is very large and most are redundant (Bayardo and Agrawal, 1999; Brin and al., 1997; Siverstein et al., 1998).

\footnotetext{
${ }^{2}$ We say that a rule $r: a \rightarrow c$ is more general than a rule $r^{\prime}: a^{\prime} \rightarrow c^{\prime}$ if they have identical supports and confidences, the antecedent $a$ of $r$ is a subset of $a^{\prime}$ and the consequent $c$ of $r$ is a superset of $c^{\prime} . r^{\prime}$ is then called a sub-rule of $r$, and $r$ a super-rule of $r^{\prime}$.
} 
With the min-max basis, the analyst is presented a set of rules covering all the attributes of the dataset: All of the data-space is characterized by the min-max rules, overcoming an important deficiency of most reduction methods where large sub-spaces of the data-space may be poorly characterized or even entirely uncharacterized (Bayardo and Agrawal, 1999). This property helps insuring that rules "surprising" for the analyst, that are important information (Piatetsky and Matheus, 1994; Silberschatz and Tuzhilin, 1996), will be present. Moreover, the min-max basis does not represent any information loss for the analyst: all information brought by the set of all association rules is brought by the min-max basis. This approach does not suffer of the problem of information loss - from the analyst's point of view - that is an important drawback in association rule reduction methods (Liu and al., 1999). If the analyst so wishes, it is also possible to efficiently deduce all other association rules, with supports and confidences, from the min-max basis alone.

\subsection{Organization}

In section 2, we recall the semantic for association rules based on the Galois connection and the CLOSE algorithm for extracting frequent closed itemsets and generators. We also present the CLOSE${ }^{+}$algorithm for efficiently deriving frequent closed itemsets, their generators and their supports from frequent itemsets and their supports. Min-max association rules and the min-max basis for association rules are defined in section 3. Algorithms for generating this basis are also presented. In section 4, we present simple methods and algorithms for deriving all association rules from the min-max basis. Results of experiments conducted to evaluate the usefulness of this approach are given in section 5 and section 6 concludes the paper.

\section{Semantic for association rules based on the Galois connection}

The association rule extraction is performed from a data mining context ${ }^{3}$, that is a triplet $\mathcal{D}=(\mathcal{O}, \mathcal{I}, \mathcal{R})$, where $\mathcal{O}$ and $\mathcal{I}$ are finite sets of objects and items respectively, and $\mathcal{R} \subseteq \mathcal{O} \times \mathcal{I}$ is a binary relation. Each couple $(o, i) \in \mathcal{R}$ denotes the fact that the object $o \in \mathcal{O}$ is related to the item $i \in \mathcal{I}$. An itemset $l$ is a set of items $l \subseteq \mathcal{I}, l \neq \varnothing$.

Example 2. A data mining context $\mathcal{D}$ constituted of six objects, each one identified by its $O I D$, and five items is represented in table II. This context is used as support for the examples in the rest of the paper.

The Galois connection of a finite binary relation (Ganter and Wille, 1999) is a couple of applications $(\phi, \psi)$. $\phi$ associates with a set of objects $O \subseteq \mathcal{O}$ the items related to all objects $o \in O$ and $\psi$ associates with an itemset $l \subseteq \mathcal{I}$ the objects related to all items $i \in l$. When an object $o$ is related to all items $i \in l$, we say that $o$ contains $l$. We denote minsupp and minconf the minimal support and confidence thresholds.

Definition 1. (Frequent itemsets) The support of an itemset $l$ is the proportion of objects in the context containing $l: \operatorname{supp}(l)=|\psi(l)| /|\mathcal{O}| . l$ is a frequent itemset if $\operatorname{supp}(l) \geq$ minsupp.

\footnotetext{
${ }^{3}$ We will use context and dataset interchangeably in the sequel.
} 
Table II. Data mining context $\mathcal{D}$.

\begin{tabular}{|ccccc|}
\hline OID & & \multicolumn{3}{c|}{ Items } \\
\hline 1 & A & C & D & \\
2 & B & C & E & \\
3 & A & B & C & E \\
4 & B & E & & \\
5 & A & B & C & E \\
6 & B & C & E & \\
\hline
\end{tabular}

Definition 2. (Association rules) An association rule $r$ is an implication between two frequent itemsets $l_{1}, l_{2} \subseteq \mathcal{I}$ with the form $l_{1} \rightarrow\left(l_{2} \backslash l_{1}\right)$ where $l_{1} \subset l_{2}$. The support and confidence of $r$ are defined by: $\operatorname{supp}(r)=\operatorname{supp}\left(l_{2}\right), \operatorname{conf}(r)=\operatorname{supp}\left(l_{2}\right) / \operatorname{supp}\left(l_{1}\right)$.

The closure operator $\gamma=\phi \circ \psi$ associates with an itemset $l$ the maximal set of items common to all the objects containing $l$ : The closure of an itemset is equal to the intersection of all the objects containing it. Using this closure operator, we define the frequent closed itemsets.

Definition 3. (Frequent closed itemsets) A frequent itemset $l \subseteq \mathcal{I}$ is a frequent closed itemset iff $\gamma(l)=l$. The minimal closed itemset containing an itemset $l$ is its closure $\gamma(l)$

The set of frequent closed itemsets and their supports is a minimal non-redundant generating set for all frequent itemsets and their supports, and thus for all association rules, their supports and their confidences. This theorem relies on the properties that the support of a frequent itemset is equal to the support of its closure and that maximal frequent itemsets are maximal frequent closed itemsets (Pasquier et al., 1998). In order to improve the efficiency of frequent closed itemset extraction, the Close and A-ClOsE algorithms compute generators of frequent closed itemsets.

Definition 4. (Generators) An itemset $g \subseteq \mathcal{I}$ is a generator of a closed itemset $l$ iff $\gamma(g)=l$ and $\nexists g^{\prime} \subseteq \mathcal{I}$ with $g^{\prime} \subset g$ such that $\gamma\left(g^{\prime}\right)=l$. A generator of cardinality $k$ is a $k$-generator.

Generators are the minimal itemsets to consider for discovering frequent closed itemsets, by computing their closures. Based on the following lemma, Close and A-CLOSE perform a breadth-first search for generators in a levelwise manner.

Lemma 1. All subsets $s \subseteq \mathcal{I}$ of a generator $g \subseteq \mathcal{I}$ are also generators. The closure of $s$ is a closed subset of the closure of $g: \gamma(s) \subset \gamma(g)$.

Proof. See (Pasquier et al., 1999b). 


\subsection{Extracting frequent Closed itemsets and generators with Close}

The CLOSE algorithm is an iterative algorithm for extracting generators and frequent closed itemsets in a levelwise manner. During an iteration $k$, a list of candidate $k$ generators is considered; their closures and their supports are computed from the dataset and infrequent generators are discarded. Frequent generators are then used to construct candidate $(k+1)$-generators. The closures of frequent generators are the frequent closed itemsets and the support of a generator is also the support of its closure.

During the $k^{\text {th }}$ iteration, a set $F C_{k}$ is considered. Each element of this set consists of three information: a $k$-generator, its closure and their support. The algorithm first initializes the candidate 1-generators in $F C_{1}$ with the list of 1-itemsets and then carries out some iterations. During each iteration $k$ :

1. Closures of all candidate $k$-generators and their supports are computed: The number of objects containing a generator determines its support and their intersection generates its closure. Each object is considered once and this phase requires only one scan of the dataset.

2. Infrequent $k$-generators, i.e., generators with support lower than minsupp, are removed from $F C_{k}$.

3. The set of candidate $(k+1)$-generators is constructed by joining the frequent $k$-generators in $F C_{k}$ as follows.

a) Two $k$-generators in $F C_{k}$ that have the same first $k-1$ items are joined to create a candidate $(k+1)$-generator. For instance, the 3 -generators $\{\mathrm{ABC}\}$ and $\{\mathrm{ABD}\}$ will be joined in order to create the candidate 4-generator $\{\mathrm{ABCD}\}$.

b) Candidate $(k+1)$-generators that are infrequent or non-minimal are removed. One of the $k$-subsets of such a generator is either infrequent or non-minimal and thus does not belong to the set of frequent $k$-generators in $F C_{k}$.

c) The third phase removes $(k+1)$-generators which closures were already computed. Such a generator $g$ is easily identified as it is included in the closure of a frequent $k$-generator $g^{\prime}$ in $F C_{k}$ : We have $g^{\prime} \subset g \subseteq \gamma\left(g^{\prime}\right)$.

The algorithm stops when no new candidate generator can be created. Then, each set $F C_{k}$ stores the frequent $k$-generators, their closures and their supports.

Example 3. Figure 1 shows the execution of the ClOSE algorithm on the context $\mathcal{D}$ for minsupp $=2 / 6$. The set $F C_{1}$ is initialized with the list of all 1 -itemsets. The algorithm computes supports and closures of the 1-generators in $F C_{1}$ and infrequent ones are discarded. Then, joining the frequent generators in $F C_{1}$, six new candidate 2-generators are created: $\{\mathrm{AB}\},\{\mathrm{AC}\},\{\mathrm{AE}\},\{\mathrm{BC}\},\{\mathrm{BE}\}$ and $\{\mathrm{CE}\}$ in $F C_{2}$. The 2-generators $\{\mathrm{AC}\}$ and $\{\mathrm{BE}\}$ are removed form $F C_{2}$ because we have $\{\mathrm{AC}\} \subseteq$ $\gamma(\{\mathrm{A}\})$ and $\{\mathrm{BE}\} \subseteq \gamma(\{\mathrm{B}\})$. The algorithm determines supports and closures of the remaining 2-generators in $F C_{2}$ and suppresses infrequent ones. Then, the candidate 3 -generator $\{\mathrm{ABE}\}$ is created by joining the frequent generators in $F C_{2}$ but is removed because the 2-generator $\{\mathrm{BE}\} \subset\{\mathrm{ABE}\}$ is not in $F C_{2}$ and the algorithm stops. 

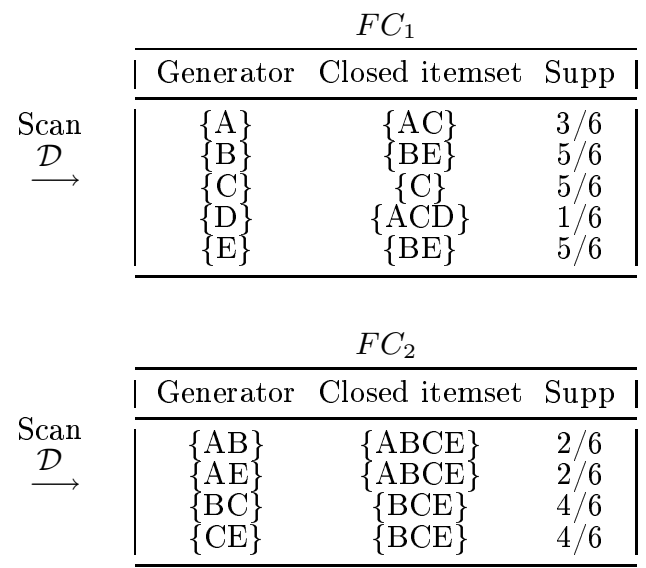
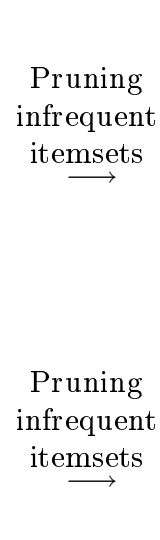

\begin{tabular}{|ccc|}
\hline \multicolumn{3}{c}{$F C_{1}$} \\
\hline Generator & Closed itemset & Supp \\
\hline A $\}$ & $\{\mathrm{AC}\}$ & $3 / 6$ \\
$\mathrm{~B}\}$ & $\{\mathrm{BE}\}$ & $5 / 6$ \\
$\mathrm{C}\}$ & $\{\mathrm{C}\}$ & $5 / 6$ \\
$\mathrm{E}\}$ & $\{\mathrm{BE}\}$ & $5 / 6$ \\
\hline
\end{tabular}

\begin{tabular}{|ccc|}
\hline Generator & Closed itemset & Supp | \\
\hline$\{\mathrm{AB}\}$ & $\{\mathrm{ABCE}\}$ & $2 / 6$ \\
AE & $\{\mathrm{ABCE}\}$ & $2 / 6$ \\
$\mathrm{BC}\}$ & $\{\mathrm{BCE}\}$ & $4 / 6$ \\
$\{\mathrm{CE}\}$ & $\{\mathrm{BCE}\}$ & $4 / 6$ \\
\hline
\end{tabular}

Figure 1. Extracting frequent closed itemsets in the context $\mathcal{D}$ with CLOSE.

The A-Close algorithm improves the efficiency of the extraction in case of weakly correlated data. It does not compute closures of candidate generators during the iterations, but during an ultimate scan carried out after the end of these iterations if necessary. Experimental results show that ClOsE and A-ClOSE are particularly efficient for mining association rules from dense or correlated data. On such data, Close outperforms A-Close, and they both outperform algorithms for extracting frequent itemsets and maximal frequent itemsets. In that case, algorithms for extracting maximal frequent itemsets suffer from the cost of the frequent itemset supports computation that requires accessing the dataset. On the contrary, for weakly correlated data, algorithms for extracting maximal frequent itemsets are the most efficient and algorithms for extracting frequent itemsets, as well as A-Close, outperform ClOSE.

The ChARM (Zaki and Hsiao, 1999) and Closet (Pei et al., 2000) algorithms extract frequent closed itemsets. However, none of these algorithm extract generators and can be used to generate the min-max basis for association rules. The PASCAL (Bastide and al., 2000) algorithm is an optimization of APRIORI based on inference counting and equivalence classes defined according to itemset supports. It can easily be extended to generate the min-max basis since generators and closed itemsets are respectively bottom and top patterns of an equivalence class.

\subsection{Deriving frequent Closed itemsets and Generators From FREQUENT ITEMSETS}

The $\mathrm{CLOSE}^{+}$algorithm identifies frequent closed itemsets and generators among frequent itemsets without accessing the dataset. It enables the efficient generation of the min-max basis when an algorithm for extracting frequent itemsets is used. Such an algorithm gives as result the sets $F_{k}$, each set $F_{k}$ containing all frequent $k$-itemsets, with $k$ varying from 1 to $\mu$ (the size of the longest maximal frequent itemsets). The frequent closed itemsets and generators are identified among frequent itemsets using propositions 1 and 2 that are derived from the property that an itemset's support is equal to its closure's support. The completeness of the approach 
is insured by the property that maximal frequent itemsets are maximal frequent closed itemsets (Pasquier et al., 1998).

Proposition 1. The support of a generator is smaller than the supports of all its subsets.

Proof. Let $g$ be a $k$-generator and $s$ a $(k-1)$-subsets of $g$. We then have $s \subset g$ $\Rightarrow \psi(s) \supseteq \psi(g)$. If $\psi(s)=\psi(g)$ then $\gamma(s)=\gamma(g)$ and $g$ is not a generator: It is not a minimal itemset whose closure is $\gamma(g)$. It follows that $\psi(s) \supset \psi(g) \Rightarrow$ $\operatorname{supp}(g)>\operatorname{supp}(s)$.

Proposition 2. The support of a closed itemset is greater than the supports of all its supersets.

Proof. Let $l$ be a closed $k$-itemset and $s$ a superset of $l$. We then have $l \subset s \Rightarrow$ $\psi(l) \supseteq \psi(s)$. If $\psi(l)=\psi(s)$ then $\gamma(l)=\gamma(s) \Rightarrow l=\gamma(s) \Rightarrow s \subseteq l$ (absurd). It follows that $\psi(l) \supset \psi(s) \Rightarrow \operatorname{supp}(l)>\operatorname{supp}(s)$.

The pseudo-code of the $\mathrm{CLOSE}^{+}$algorithm is given in figure 2. It examines successively all frequent itemsets in each set $F_{k}$, with $k$ varying from 1 to $\mu$. It generates the sets $F C_{m}, 1 \leq m \leq \nu$, where $\nu$ is the size of the longest generators, containing the $m$-generators, their closures and their supports. It first determines if a frequent $k$ itemset is a generator by examining all its $(k-1)$-subsets' supports; it then determine if it is a closed itemset by examining all its $(k+1)$-supersets' supports and if so, identifies its generators by examining all its subsets' supports. The boolean variables isclosed and isgenerator are used to determine if an itemset $l$ is a closed itemset or is a generator.

At the beginning of the $k^{t h}$ iteration (steps 1 to 21 ), the set $F C_{k}$ is empty (step 2). In steps 3 to 20, frequent itemsets in $F_{k}$ are considered successively. If an itemset $l$ has the same support as one of its $(k-1)$-subset $l^{\prime}$ in $F_{k-1}$ (steps 5 to 7 ), then $l$ is not a generator (step 6). Otherwise, $l$ and its support are inserted in $F C_{k}$ (step 8). Then, we test if $l$ has the same support as one of its $(k+1)$-superset $l$ " in $F_{k+1}$ (steps 10 to 12). If so, we have $l^{\prime} \subseteq \gamma(l)$ and then $l \neq \gamma(l): l$ is not closed (step 11). Otherwise, $l$ is a frequent closed itemset and we determine the generators of $l$ (steps 13 to 19) as follows. For each generator $g$ of size $n$, with $1 \leq n \leq k$, that is a subset of $l$ (steps 14 to 18), if the supports of $g$ and $l$ are equal then $g$ is a generator of $l$ and $l$ is inserted in $F C_{n}$ as the closure of $g$ (step 16). Thus, at the end of the algorithm, each set $F C_{k}$ contains all frequent $k$-generators, their closures and their supports.

Correctness. The correctness of the computation of sets $F C_{k}$ for $1 \leq k \leq \mu$ relies on propositions 1 and 2. Using the first one, we determine if a frequent $k$-itemset $l$ is a generator of a closed itemset by comparing its support and the supports of the frequent $(k-1)$-itemsets included in $l$. The second proposition enables to determine if a frequent $k$-itemset $l$ is closed by comparing its support and the supports of the frequent $(k+1)$-itemsets in which $l$ is included. Since a generator has the same support as its closure, the determination of the generators of a closed itemset is correct. 


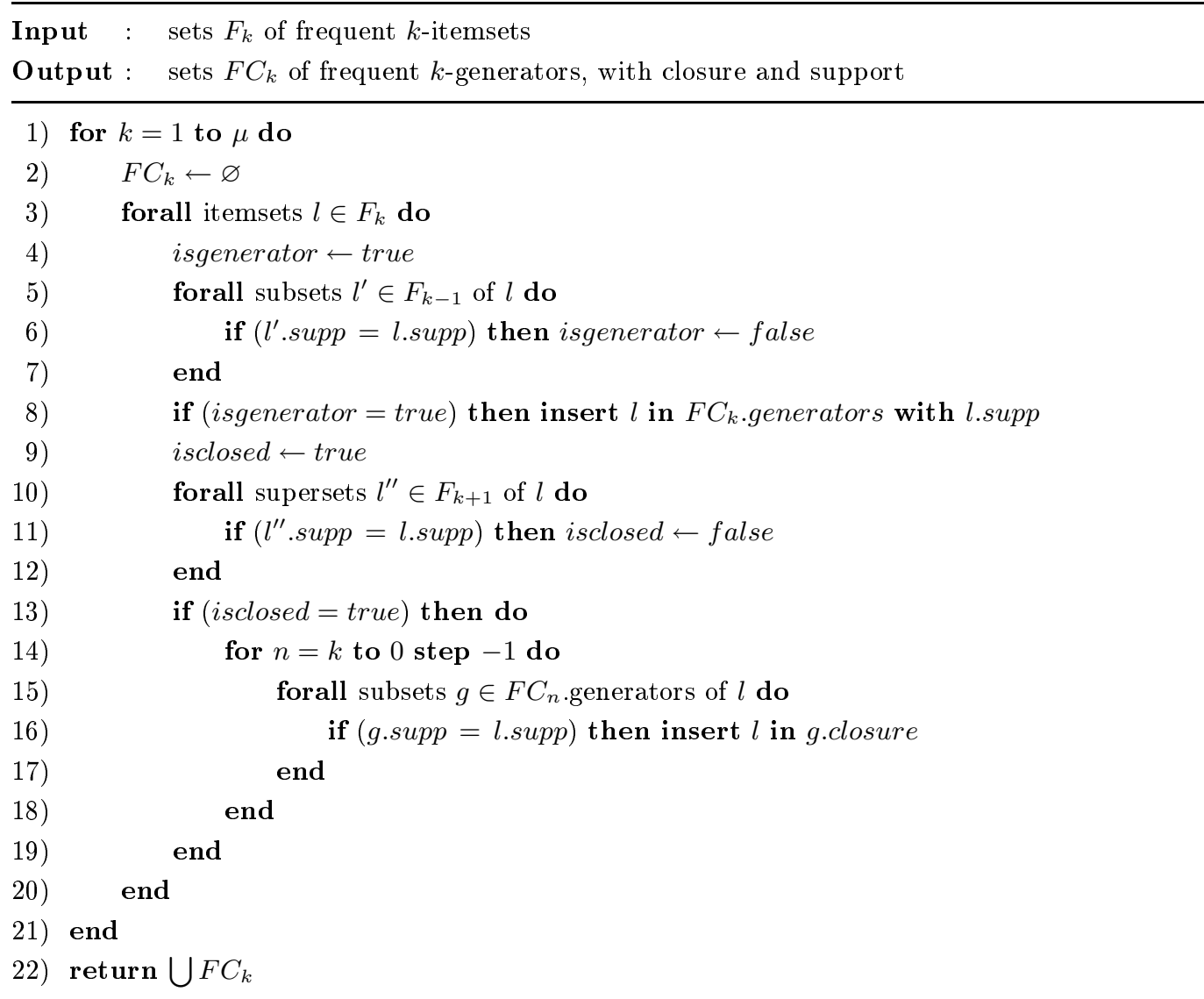

Figure 2. $\mathrm{CLOSE}^{+}$algorithm for deriving frequent closed itemsets and generators.

Example 4. Figure 3 shows the execution of the $\mathrm{CLOSE}^{+}$algorithm using the sets $F_{1}$ to $F_{4}$ of frequent itemsets extracted from the context $\mathcal{D}$ with minsupp $=2 / 6$. All frequent 1-itemsets are frequent 1 -generators since none of their subsets is a frequent itemset: The empty set is not considered as a frequent itemset. The 1-itemset $\{\mathrm{C}\}$ is also its own closure since all its supersets in $F_{2}$ have a smaller support. In $F_{2}$, the 2-itemsets $\{\mathrm{AC}\}$ and $\{\mathrm{BE}\}$ are not generators since they have the same support as itemsets $\{A\}$ and, $\{B\}$ and $\{E\}$ respectively. These two itemsets are closed since their support is lower than those of all their supersets in $F_{3} ;\{\mathrm{AC}\}$ is the closure of $\{\mathrm{A}\}$ and $\{\mathrm{BE}\}$ is the closure of $\{\mathrm{B}\}$ and $\{\mathrm{E}\}$. No frequent 3-itemset in $F_{3}$ is a generator and $\{\mathrm{BCE}\}$, that has the same support as $\{\mathrm{BC}\}$ and $\{\mathrm{CE}\}$ and a greater support than $\{\mathrm{ABCE}\}$ in $F C_{4}$, is the closure of $\{\mathrm{BC}\}$ and $\{\mathrm{CE}\}$ in $F C_{2}$. Finally, the 4 -itemset $\{\mathrm{ABCE}\}$ is closed since it is a maximal frequent itemset is the closure of $\{\mathrm{AB}\}$ and $\{\mathrm{AE}\}$, and is inserted in $F C_{2}$.

Remark. As a simple optimization, the algorithm can stop testing if frequent $k$ itemsets are generators after the first iteration $n$ during which no frequent $n$-itemset examined is a generator. In example 4 , the algorithm will not test if 4 -itemsets in $F_{4}$ are generators since no 3 -itemset is a generator $\left(F C_{3}\right.$ is empty at the end of the third iteration). 


\begin{tabular}{cc}
\multicolumn{2}{c}{$F_{1}$} \\
\hline Itemset & Supp \\
\hline$\{\mathrm{A}\}$ & $3 / 6$ \\
$\mathrm{~B}\}$ & $5 / 6$ \\
$\mathrm{C}\}$ & $5 / 6$ \\
$\mathrm{E}\}$ & $5 / 6$ \\
\hline
\end{tabular}

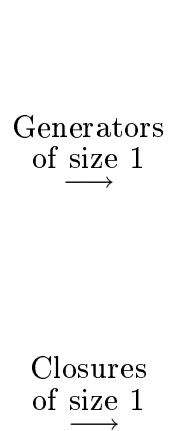

\begin{tabular}{|c|c|c|}
\hline \multicolumn{3}{|c|}{$F C_{1}$} \\
\hline Generator & Closed itemset & Supp \\
\hline$\left\{\begin{array}{l}\mathrm{A}\} \\
\mathrm{B} \\
\mathrm{C} \\
\mathrm{E}\}\end{array}\right.$ & & $\begin{array}{l}3 / 6 \\
5 / 6 \\
5 / 6 \\
5 / 6\end{array}$ \\
\hline \multicolumn{3}{|c|}{$F C_{1}$} \\
\hline Generator & Closed itemset & Supp \\
\hline 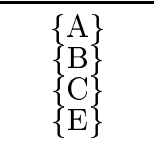 & $\{C\}$ & $\begin{array}{l}3 / 6 \\
5 / 6 \\
5 / 6 \\
5 / 6\end{array}$ \\
\hline
\end{tabular}

\begin{tabular}{cc}
\multicolumn{2}{c}{$F_{2}$} \\
\hline Itemset & Supp \\
\hline$\{\mathrm{AB}\}$ & $2 / 6$ \\
$\mathrm{AC}\}$ & $3 / 6$ \\
$\mathrm{AE}\}$ & $2 / 6$ \\
$\mathrm{BC}\}$ & $4 / 6$ \\
BE & $5 / 6$ \\
CE & $4 / 6$ \\
\hline
\end{tabular}

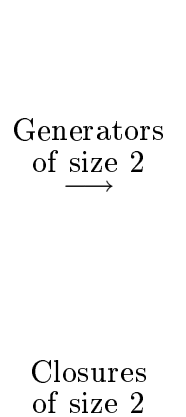

\begin{tabular}{|c|c|c|}
\hline \multicolumn{3}{|c|}{$F C_{2}$} \\
\hline Generator & Closed itemset & Supp \\
\hline$\{\mathrm{AB}\}$ & & $2 / 6$ \\
\hline AE & & $2 / 6$ \\
\hline$\{\mathrm{BC}\}$ & & $4 / 6$ \\
\hline$\{\mathrm{CE}\}$ & & $4 / 6$ \\
\hline \multicolumn{3}{|c|}{$F C_{1}$} \\
\hline Generator & Closed itemset & Supp \\
\hline$\{\mathrm{A}\}$ & $\{\mathrm{AC}\}$ & $3 / 6$ \\
\hline B $\}$ & $\{\mathrm{BE}\}$ & $5 / 6$ \\
\hline$\{\mathrm{C}\}$ & $\{C\}$ & $5 / 6$ \\
\hline$\{\mathrm{E}\}$ & $\{\mathrm{BE}\}$ & $5 / 6$ \\
\hline
\end{tabular}

\begin{tabular}{cc}
\multicolumn{2}{c}{$F_{3}$} \\
\hline Itemset & Supp \\
\hline$\{$ ABC & $2 / 6$ \\
ABE & $2 / 6$ \\
ACE & $2 / 6$ \\
BCE $\}$ & $4 / 6$ \\
\hline
\end{tabular}

Generators of size 3

\begin{tabular}{ccc}
\hline \multicolumn{2}{c}{$F C_{3}$} \\
\hline Generator & Closed itemset & Supp \\
\hline & $F C_{2}$ \\
\hline Generator & Closed itemset & Supp \\
\hline$\{$ AB & & $2 / 6$ \\
AE \{ & & $2 / 6$ \\
BC $\}$ & $\{$ BCE $\}$ & $4 / 6$ \\
CE $\}$ & $\{$ BCE $\}$ & $4 / 6$ \\
\hline
\end{tabular}

Closures of size 3
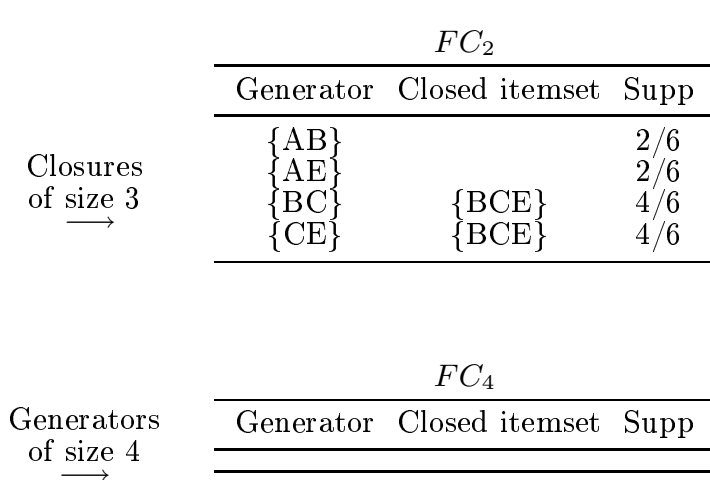

\begin{tabular}{cc}
\multicolumn{2}{c}{$F_{4}$} \\
\hline Itemset & Supp \\
\hline$\{$ ABCE $\}$ & $2 / 6$ \\
\hline
\end{tabular}
of $\stackrel{\text { size } 4}{\longrightarrow}$

\begin{tabular}{ccc}
\multicolumn{3}{c}{$F C_{2}$} \\
\hline Generator & Closed itemset & Supp \\
\hline$\{\mathrm{AB}\}$ & $\{$ ABCE & $2 / 6$ \\
AE $\}$ & ABCE $\}$ & $2 / 6$ \\
BC & $\{$ BCE & $4 / 6$ \\
CE $\}$ & $\{$ BCE $\}$ & $4 / 6$ \\
\hline
\end{tabular}

Figure 3. Deriving frequent closed itemsets and generators with CLOSE ${ }^{+}$. 


\section{Min-max basis for association rules}

We first define min-max association rules: The most general non-redundant association rules according to their semantic. Informally, an association rule is redundant if it brings the same information or less information than is brought by another rule of same support and confidence. Then, the min-max association rules are the nonredundant association rules having minimal antecedent and maximal consequent: $r$ is a min-max association rule if no other association rule $r^{\prime}$ has the same support and confidence, an antecedent that is a subset of the antecedent of $r$ and a consequent that is a superset of the consequent of $r$.

Definition 5. (Min-max association rules) Let $\mathcal{A} R$ be the set of association rules extracted. An association rule $r: l_{1} \rightarrow l_{2} \in \mathcal{A} R$ is a min-max association rule iff $\nexists r^{\prime}: l_{1}^{\prime} \rightarrow l_{2}^{\prime} \in \mathcal{A} R$ with $\operatorname{supp}\left(r^{\prime}\right)=\operatorname{supp}(r), \operatorname{conf}\left(r^{\prime}\right)=\operatorname{conf}(r), l_{1}^{\prime} \subseteq l_{1}$ and $l_{2} \subseteq l_{2}^{\prime}$.

Based on this definition, we characterize exact and approximate min-max association rules that constitute respectively the min-max exact basis and the min-max approximate basis in the two following sections.

\subsection{EXACT MIN-MAX ASSOCIATION RULES}

First, notice that exact association rules, with the form $r: l_{1} \Rightarrow\left(l_{2} \backslash l_{1}\right)$, are rules between two frequent itemsets $l_{1} \subset l_{2}$ having the same closure: $\gamma\left(l_{1}\right)=\gamma\left(l_{2}\right)$. Since $\operatorname{conf}(r)=1$ we have $\operatorname{supp}\left(l_{1}\right)=\operatorname{supp}\left(l_{2}\right)$, and as $l_{1} \subset l_{2}$ we see that $\gamma\left(l_{1}\right)=\gamma\left(l_{2}\right)$. We define min-max association rules among these exact rules.

Let $g$ be the generator of $\gamma\left(l_{1}\right)=\gamma\left(l_{2}\right)$ such that $g \subseteq l_{1}$. Since $g$ is minimal, we have $g \subseteq l_{1} \subset l_{2} \subseteq \gamma\left(l_{2}\right)$. Furthermore, all itemsets in the interval $\left[g, \gamma\left(l_{2}\right)\right]$, defined by inclusion ${ }^{4}$, have the same closure $\gamma\left(l_{2}\right)$ and thus the same support. The min-max association rule among all rules with the form $r: l_{1} \Rightarrow\left(l_{2} \backslash l_{1}\right)$ with $l_{1}, l_{2} \in\left[g, \gamma\left(l_{2}\right)\right]$ is the rule $g \Rightarrow\left(\gamma\left(l_{2}\right) \backslash g\right)$. This rule has a minimal antecedent, $g$, and a maximal consequent, $\gamma\left(l_{2}\right)$, among all these rules that have the same support.

We generalize this definition to all generators of the frequent closed itemset $\gamma\left(l_{2}\right)$. Let $G e n_{\gamma\left(l_{2}\right)}$ be the set of these generators. All exact min-max association rules constructed with $\gamma\left(l_{2}\right)$ are rules with the form $g \Rightarrow\left(\gamma\left(l_{2}\right) \backslash g\right)$ with $g \in G e n_{\gamma\left(l_{2}\right)}$. The extension of this property to all frequent closed itemsets defines the min-max exact basis containing all exact min-max association rules characterized in definition 5 .

Definition 6. (Min-max exact basis) Let Closed be the set of frequent closed itemsets extracted from the context and, for each frequent closed itemset $f$, let's denote $\operatorname{Gen}_{f}$ the set of generators of $f$. The min-max exact basis is:

$$
\text { MinMaxExact }=\left\{r: g \Rightarrow(f \backslash g) \mid f \in \text { Closed } \wedge g \in \text { Gen }_{f} \wedge g \neq f\right\} \text {. }
$$

The condition $g \neq f$ discards rules with the form $g \Rightarrow \varnothing$; it is equivalent to the condition $l_{1} \subset l_{2}$ in the definition of association rules. We state in the following proposition that the min-max exact basis does not lead to information loss.

\footnotetext{
${ }^{4}$ The interval $\left[l_{1}, l_{2}\right]$ contains all the supersets of $l_{1}$ that are subsets of $l_{2}$.
} 
The pseudo-code of the algorithm for constructing the min-max exact basis using frequent closed itemsets and their generators is presented in figure 4. Each element of a set $F C_{k}$ contains three fields: a $k$-generator generator, its closure closure and their support supp. The algorithm returns the set MinMaxExact containing the exact min-max rules.

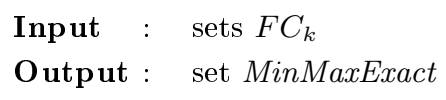

1) MinMaxExact $\leftarrow \varnothing$

2) for $k=1$ to $\nu$ do

3) forall $k$-generator $g \in F C_{k}$ do

4) if $(g \neq$ g.closure)

5) then insert $\{r: g \Rightarrow$ (g.closure $\backslash g)$, g.supp $\}$ in MinMaxExact

6) end

7) end

8) return MinMaxExact

Figure 4. Algorithm for generating the min-max exact basis.

First, MinMaxExact is initialized with the empty set (step 1). Then, each set $F C_{k}$ is examined in increasing order of $k$ values (steps 2 to 7 ). For each $k$-generator $g \in F C_{k}$ of the frequent closed itemset $\gamma(g)$ (steps 3 to 6), if $g$ is different from its closure $\gamma(g)$ (step 4), the rule $r: g \Rightarrow(\gamma(g) \backslash g)$, which support is equal to the support of $g$ and $\gamma(g)$, is inserted into MinMaxExact (step 5). Finally, the algorithm returns the set MinMaxExact containing all exact min-max association rules between generators and their closures (step 8).

Example 5. The min-max exact basis extracted from context $\mathcal{D}$ for minsupp $=2 / 6$ is presented in table III. It contains seven rules whereas the set of all exact association rules, presented in table IV, contains fourteen rules.

Table III. Min-max exact basis extracted from $\mathcal{D}$.

\begin{tabular}{|llll|}
\hline Generator & Closure & Exact rule & Supp \\
\hline$\{\mathrm{A}\}$ & $\{\mathrm{AC}\}$ & $\mathrm{A} \Rightarrow \mathrm{C}$ & $3 / 6$ \\
$\{\mathrm{~B}\}$ & $\{\mathrm{BE}\}$ & $\mathrm{B} \Rightarrow \mathrm{E}$ & $5 / 6$ \\
$\{\mathrm{C}\}$ & $\{\mathrm{C}\}$ & & \\
$\{\mathrm{E}\}$ & $\{\mathrm{BE}\}$ & $\mathrm{E} \Rightarrow \mathrm{B}$ & $5 / 6$ \\
$\{\mathrm{AB}\}$ & $\{\mathrm{ABCE}\}$ & $\mathrm{AB} \Rightarrow \mathrm{CE}$ & $2 / 6$ \\
$\{\mathrm{AE}\}$ & $\{\mathrm{ABCE}\}$ & $\mathrm{AE} \Rightarrow \mathrm{BC}$ & $2 / 6$ \\
$\{\mathrm{BC}\}$ & $\{\mathrm{BCE}\}$ & $\mathrm{BC} \Rightarrow \mathrm{E}$ & $4 / 6$ \\
$\{\mathrm{CE}\}$ & $\{\mathrm{BCE}\}$ & $\mathrm{CE} \Rightarrow \mathrm{B}$ & $4 / 6$ \\
\hline
\end{tabular}


Table IV. Exact association rules extracted from $\mathcal{D}$.

\begin{tabular}{|cc|cc|}
\hline Exact rule & Supp & Exact rule & Supp \\
\hline $\mathrm{A} \Rightarrow \mathrm{C}$ & $3 / 6$ & $\mathrm{BC} \Rightarrow \mathrm{E}$ & $4 / 6$ \\
$\mathrm{~B} \Rightarrow \mathrm{E}$ & $5 / 6$ & $\mathrm{CE} \Rightarrow \mathrm{B}$ & $4 / 6$ \\
$\mathrm{E} \Rightarrow \mathrm{B}$ & $5 / 6$ & $\mathrm{AB} \Rightarrow \mathrm{CE}$ & $2 / 6$ \\
$\mathrm{AB} \Rightarrow \mathrm{C}$ & $2 / 6$ & $\mathrm{AE} \Rightarrow \mathrm{BC}$ & $2 / 6$ \\
$\mathrm{AB} \Rightarrow \mathrm{E}$ & $2 / 6$ & $\mathrm{ABC} \Rightarrow \mathrm{E}$ & $2 / 6$ \\
$\mathrm{AE} \Rightarrow \mathrm{B}$ & $2 / 6$ & $\mathrm{ABE} \Rightarrow \mathrm{C}$ & $2 / 6$ \\
$\mathrm{AE} \Rightarrow \mathrm{C}$ & $2 / 6$ & $\mathrm{ACE} \Rightarrow \mathrm{B}$ & $2 / 6$ \\
\hline
\end{tabular}

Proposition 3. (i) All exact association rules and their supports can be deduced from the min-max exact basis. (ii) All rules in the min-max exact basis are min-max association rules.

Proof. (i) Let $r: l_{1} \Rightarrow\left(l_{2} \backslash l_{1}\right)$ be an exact association rule between two frequent itemsets with $l_{1} \subset l_{2}$. Since conf $(r)=1$, we have $\operatorname{supp}\left(l_{1}\right)=\operatorname{supp}\left(l_{2}\right)$ and as an itemset's support is equal to its closure's support, we deduce that $\operatorname{supp}\left(\gamma\left(l_{1}\right)\right)=$ $\operatorname{supp}\left(\gamma\left(l_{2}\right)\right)$ which implies that $\gamma\left(l_{1}\right)=\gamma\left(l_{2}\right)=f$. The itemset $f$ is a frequent closed itemset $f \in F C$ and, obviously, there exists a rule $r^{\prime}: g \Rightarrow(f \backslash g) \in$ MinMaxExact such that $g$ is a generator of $f$ with $g \subseteq l_{1}$ and $g \subset l_{2}$. We show now that the rule $r$ and its support can be deduced from the rule $r^{\prime}$ and its support. Since $g \subseteq l_{1} \subset l_{2} \subseteq$ $f$, rule $r$ 's antecedent and consequent can be derived from those of rule $r^{\prime}$. From $\gamma\left(l_{1}\right)=\gamma\left(l_{2}\right)=f$, we deduce that $\operatorname{supp}(r)=\operatorname{supp}\left(l_{2}\right)=\operatorname{supp}\left(\gamma\left(l_{2}\right)\right)=\operatorname{supp}(f)=$ $\operatorname{supp}\left(r^{\prime}\right)$.

(ii) Let $r: g \Rightarrow(f \backslash g) \in$ MinMaxExact. According to definition 6, we have $g \in G n_{f}$ and $f \in$ Closed. We demonstrate that there is no other rule $r^{\prime}: l_{1}^{\prime} \Rightarrow\left(l_{2}^{\prime} \backslash l_{1}^{\prime}\right) \in$ MinMaxExact, such as $\operatorname{supp}\left(r^{\prime}\right)=\operatorname{supp}(r), \operatorname{conf}\left(r^{\prime}\right)=\operatorname{conf}(r), l_{1}^{\prime} \subseteq g$ and $f \subseteq l_{2}^{\prime}$. If $l_{1}^{\prime} \subset g$ then, according to definition 4, we have $\gamma\left(l_{1}^{\prime}\right) \subset \gamma(g)=f \Longrightarrow l_{1} \notin G e n_{f}$ and then $r^{\prime} \notin$ MinMaxExact. If $f \subset l_{2}^{\prime}$ and according to definition 3, we have $f=\gamma(f)=\gamma(g) \subset l_{2}^{\prime}=\gamma\left(l_{2}^{\prime}\right)$. From definition 4 we deduce $g \notin G e n_{l_{2}^{\prime}}$ and we conclude that $r^{\prime} \notin$ MinMaxExact.

\subsection{Approximate min-maX association Rules}

Approximate association rules, with the form $r: l_{1} \rightarrow\left(l_{2} \backslash l_{1}\right)$, are rules between two frequent itemsets $l_{1} \subset l_{2}$ such that $\gamma\left(l_{1}\right) \subset \gamma\left(l_{2}\right)$. Since $\operatorname{conf}(r)<1$ we have $\operatorname{supp}\left(l_{1}\right)>\operatorname{supp}\left(l_{2}\right)$ and we deduce that $\gamma\left(l_{1}\right) \subset \gamma\left(l_{2}\right)$.

We deduce the definition of approximate min-max association rules. Let $g_{1}$ be a generator of the frequent closed itemset $f_{1}$ and $g_{2}$ be a generator of the frequent closed itemset $f_{2}$ such that $f_{1} \subset g_{2} \subseteq l_{2} \subseteq f_{2}$. All rules with the form $r: l_{1} \rightarrow\left(l_{2} \backslash l_{1}\right)$ where $l_{1} \in\left[g_{1}, f_{1}\right]$ and $l_{2} \in\left[g_{2}, f_{2}\right]$ have the same confidence and the same support since $g_{1}, l_{1}$ and $f_{1}$ have the same support as well as $g_{2}, l_{2}$ and $f_{2}$. We then deduce that the min-max association rule among all these rules is $g_{1} \rightarrow\left(f_{2} \backslash g_{1}\right)$. Indeed, $g_{1}$ is the minimal itemset in $\left[g_{1}, f_{1}\right]$ and $f_{2}$ is the maximal itemset in $\left[g_{2}, f_{2}\right]$. 
The generalization of this property to all couples of frequent itemsets $l_{1}$ and $l_{2}$ such that $l_{1} \subset l_{2}$ and $\operatorname{supp}\left(l_{1}\right) \neq \operatorname{supp}\left(l_{2}\right)$ defines the min-max approximate basis containing all approximate min-max association rules characterized in definition 5 .

Definition 7. (Min-max approximate basis) We denote Gen the set of generators of the frequent closed itemsets in Closed. The min-max approximate basis is:

$$
\text { MinMaxApprox }=\{r: g \rightarrow(f \backslash g) \mid f \in \text { Closed } \wedge g \in \text { Gen } \wedge \gamma(g) \subset f\} .
$$

The pseudo code of the algorithm for generating the set MinMaxApprox of approximate min-max rules using frequent closed itemsets and their generators is presented in figure 5 .

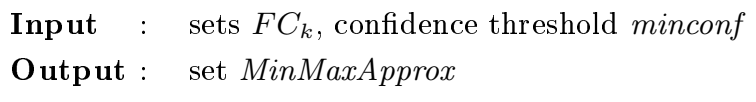

1) MinMaxApprox $\leftarrow \varnothing$

2) for $k=1$ to $\nu-1$ do

3) forall $k$-generator $g \in F C_{k}$ do

4) forall frequent closed itemset $f \in F_{j>k} \mid f \supset$ g.closure do

5) if (f.supp/g.supp $\geq$ minconf $)$

6) then insert $\{r: g \rightarrow(f \backslash g)$, f.supp/g.supp, f.supp $\}$ in MinMaxApprox

7) end

8) end

9) end

10) return MinMaxApprox

Figure 5. Algorithm for generating the min-max approximate basis.

The algorithm examines the sets $F C_{k}$ in increasing order of $k$ values (steps 2 to 9 ). For each $k$-generator $g \in F C_{k}$ (steps 3 to 8 ), it considers all closed supersets $f$ of the closure of $g$ (steps 4 to 7 ). It computes the confidence of the rule $r: g \rightarrow(f \backslash g)$ (step 5) and inserts $r$ in MinMaxReduc if it is above the minconf threshold (step 6).

Example 6. The min-max approximate basis extracted from context $\mathcal{D}$ for minsupp $=2 / 6$ and $\operatorname{minconf}=2 / 5$ is presented in table $\mathrm{V}$. It contains ten rules whereas the set of all approximate association rules, presented in table VI, contains thirty-six rules.

Proposition 4. (i) All approximate association rules can be deduced, with their supports and confidences, from the min-max approximate basis. (ii) All rules in the min-max approximate basis are min-max association rules.

Proof. (i) Let $r: l_{1} \rightarrow\left(l_{2} \backslash l_{1}\right)$ be an association rule between two frequent itemsets with $l_{1} \subset l_{2}$. Since $\operatorname{conf}(r)<1$ we also have $\gamma\left(l_{1}\right) \subset \gamma\left(l_{2}\right)$. For any frequent itemsets $l_{1}$ and $l_{2}$, there is a generator $g_{1}$ such that $g_{1} \subset l_{1} \subseteq \gamma\left(l_{1}\right)=\gamma\left(g_{1}\right)$ and a generator $g_{2}$ such that $g_{2} \subset l_{2} \subseteq \gamma\left(l_{2}\right)=\gamma\left(g_{2}\right)$. Since $l_{1} \subset l_{2}$, we have $l_{1} \subseteq \gamma\left(g_{1}\right) \subset l_{2} \subseteq \gamma\left(g_{2}\right)$ and the rule $r^{\prime}: g_{1} \rightarrow\left(\gamma\left(g_{2}\right) \backslash g_{1}\right)$ is in the min-max approximate basis. We show 
Table V. Min-max approximate basis extracted from $\mathcal{D}$.

\begin{tabular}{|lllccc|}
\hline Generator & Closure & Closed superset & Approximate rule & Supp & Conf \\
\hline$\{\mathrm{A}\}$ & $\{\mathrm{AC}\}$ & $\{\mathrm{ABCE}\}$ & $\mathrm{A} \rightarrow \mathrm{BCE}$ & $2 / 6$ & $2 / 3$ \\
$\{\mathrm{~B}\}$ & $\{\mathrm{BE}\}$ & $\{\mathrm{BCE}\}$ & $\mathrm{B} \rightarrow \mathrm{CE}$ & $4 / 6$ & $4 / 5$ \\
$\{\mathrm{~B}\}$ & $\{\mathrm{BE}\}$ & $\{\mathrm{ABCE}\}$ & $\mathrm{B} \rightarrow \mathrm{ACE}$ & $2 / 6$ & $2 / 5$ \\
$\{\mathrm{C}\}$ & $\{\mathrm{C}\}$ & $\{\mathrm{AC}\}$ & $\mathrm{C} \rightarrow \mathrm{A}$ & $3 / 6$ & $3 / 5$ \\
$\{\mathrm{C}\}$ & $\{\mathrm{C}\}$ & $\{\mathrm{BCE}\}$ & $\mathrm{C} \rightarrow \mathrm{BE}$ & $4 / 6$ & $4 / 5$ \\
$\{\mathrm{C}\}$ & $\{\mathrm{C}\}$ & $\{\mathrm{ABCE}\}$ & $\mathrm{C} \rightarrow \mathrm{ABE}$ & $2 / 6$ & $2 / 5$ \\
$\{\mathrm{E}\}$ & $\{\mathrm{BE}\}$ & $\{\mathrm{BCE}\}$ & $\mathrm{E} \rightarrow \mathrm{BC}$ & $4 / 6$ & $4 / 5$ \\
$\{\mathrm{E}\}$ & $\{\mathrm{BE}\}$ & $\{\mathrm{ABCE}\}$ & $\mathrm{E} \rightarrow \mathrm{ABC}$ & $2 / 6$ & $2 / 5$ \\
$\{\mathrm{AB}\}$ & $\{\mathrm{ABCE}\}$ & & & & \\
$\{\mathrm{AE}\}$ & $\{\mathrm{ABCE}\}$ & & $\mathrm{BC} \rightarrow \mathrm{AE}$ & $2 / 6$ & $2 / 4$ \\
$\{\mathrm{BC}\}$ & $\{\mathrm{BCE}\}$ & $\{\mathrm{ABCE}\}$ & $\mathrm{CE} \rightarrow \mathrm{AB}$ & $2 / 6$ & $2 / 4$ \\
$\{\mathrm{CE}\}$ & $\{\mathrm{BCE}\}$ & $\{\mathrm{ABCE}\}$ & & & \\
\hline
\end{tabular}

Table VI. Approximate association rules extracted from $\mathcal{D}$.

\begin{tabular}{|ccc|ccc|ccc|}
\hline Approximate rule & Supp & Conf & Approximate rule & Supp & Conf & Approximate rule & Supp & Conf \\
\hline $\mathrm{BCE} \rightarrow \mathrm{A}$ & $2 / 6$ & $2 / 4$ & $\mathrm{~B} \rightarrow \mathrm{ACE}$ & $2 / 6$ & $2 / 5$ & $\mathrm{~B} \rightarrow \mathrm{CE}$ & $4 / 6$ & $4 / 5$ \\
$\mathrm{AC} \rightarrow \mathrm{BE}$ & $2 / 6$ & $2 / 3$ & $\mathrm{C} \rightarrow \mathrm{ABE}$ & $2 / 6$ & $2 / 5$ & $\mathrm{C} \rightarrow \mathrm{BE}$ & $4 / 6$ & $4 / 5$ \\
$\mathrm{BC} \rightarrow \mathrm{AE}$ & $2 / 6$ & $2 / 4$ & $\mathrm{E} \rightarrow \mathrm{ABC}$ & $2 / 6$ & $2 / 5$ & $\mathrm{E} \rightarrow \mathrm{BC}$ & $4 / 6$ & $4 / 5$ \\
$\mathrm{BE} \rightarrow \mathrm{AC}$ & $2 / 6$ & $2 / 5$ & $\mathrm{~A} \rightarrow \mathrm{BC}$ & $2 / 6$ & $2 / 3$ & $\mathrm{~A} \rightarrow \mathrm{B}$ & $2 / 6$ & $2 / 3$ \\
$\mathrm{CE} \rightarrow \mathrm{AB}$ & $2 / 6$ & $2 / 4$ & $\mathrm{~B} \rightarrow \mathrm{AC}$ & $2 / 6$ & $2 / 5$ & $\mathrm{~B} \rightarrow \mathrm{A}$ & $2 / 6$ & $2 / 5$ \\
$\mathrm{AC} \rightarrow \mathrm{B}$ & $2 / 6$ & $2 / 3$ & $\mathrm{C} \rightarrow \mathrm{AB}$ & $2 / 6$ & $2 / 5$ & $\mathrm{C} \rightarrow \mathrm{A}$ & $3 / 6$ & $3 / 5$ \\
$\mathrm{BC} \rightarrow \mathrm{A}$ & $2 / 6$ & $2 / 4$ & $\mathrm{~A} \rightarrow \mathrm{BE}$ & $2 / 6$ & $2 / 3$ & $\mathrm{~A} \rightarrow \mathrm{E}$ & $2 / 6$ & $2 / 3$ \\
$\mathrm{BE} \rightarrow \mathrm{A}$ & $2 / 6$ & $2 / 5$ & $\mathrm{~B} \rightarrow \mathrm{AE}$ & $2 / 6$ & $2 / 5$ & $\mathrm{E} \rightarrow \mathrm{A}$ & $2 / 6$ & $2 / 5$ \\
$\mathrm{AC} \rightarrow \mathrm{E}$ & $2 / 6$ & $2 / 3$ & $\mathrm{E} \rightarrow \mathrm{AB}$ & $2 / 6$ & $2 / 5$ & $\mathrm{~B} \rightarrow \mathrm{C}$ & $4 / 6$ & $4 / 5$ \\
$\mathrm{CE} \rightarrow \mathrm{A}$ & $2 / 6$ & $2 / 4$ & $\mathrm{~A} \rightarrow \mathrm{CE}$ & $2 / 6$ & $2 / 3$ & $\mathrm{C} \rightarrow \mathrm{B}$ & $4 / 6$ & $4 / 5$ \\
$\mathrm{BE} \rightarrow \mathrm{C}$ & $4 / 6$ & $4 / 5$ & $\mathrm{C} \rightarrow \mathrm{AE}$ & $2 / 6$ & $2 / 5$ & $\mathrm{C} \rightarrow \mathrm{E}$ & $4 / 6$ & $4 / 5$ \\
$\mathrm{~A} \rightarrow \mathrm{BCE}$ & $2 / 6$ & $2 / 3$ & $\mathrm{E} \rightarrow \mathrm{AC}$ & $2 / 6$ & $2 / 5$ & $\mathrm{E} \rightarrow \mathrm{C}$ & $4 / 6$ & $4 / 5$ \\
\hline
\end{tabular}

that the rule $r$, its support and its confidence can be deduced from the rule $r^{\prime}$, its support and its confidence. Since $g_{1} \subset l_{1} \subseteq \gamma\left(g_{1}\right) \subset g_{2} \subset l_{2} \subseteq \gamma\left(g_{2}\right)$, the antecedent and the consequent of $r$ can be rebuilt starting from the rule $r^{\prime}$. Moreover, we have $\gamma\left(l_{2}\right)=\gamma\left(g_{2}\right)$ and thus $\operatorname{supp}(r)=\operatorname{supp}\left(l_{2}\right)=\operatorname{supp}\left(\gamma\left(g_{2}\right)\right)=\operatorname{supp}\left(r^{\prime}\right)$. Since $g_{1} \subset l_{1} \subseteq \gamma\left(g_{1}\right)$, we have $\operatorname{supp}\left(g_{1}\right)=\operatorname{supp}\left(l_{1}\right)$ and we thus deduce that: $\operatorname{conf}(r)=$ $\operatorname{supp}\left(l_{1}\right) / \operatorname{supp}\left(l_{2}\right)=\operatorname{supp}\left(g_{1}\right) / \operatorname{supp}\left(\gamma\left(g_{2}\right)\right)=\operatorname{conf}\left(r^{\prime}\right)$.

(ii) Let $r: g \Rightarrow(f \backslash g) \in$ MinMaxExact. According to definition 7, we have $f \in$ Closed, $g \in$ Gen $_{f^{\prime}}$ and $f^{\prime} \subset f$. We demonstrate that there is no other rule $r^{\prime}: l_{1}^{\prime} \Rightarrow$ $\left(l_{2}^{\prime} \backslash l_{1}^{\prime}\right) \in$ MinMaxApprox, such as $\operatorname{supp}\left(r^{\prime}\right)=\operatorname{supp}(r), \operatorname{conf}\left(r^{\prime}\right)=\operatorname{conf}(r), l_{1}^{\prime} \subseteq g$ and $f \subseteq l_{2}^{\prime}$. If $l_{1}^{\prime} \subset g$ then, according to definition 4 , we have $\gamma\left(l_{1}^{\prime}\right) \subset \gamma(g)=f^{\prime}$ and then $l_{1} \notin \operatorname{Gen}_{f^{\prime}}$. We deduce that $\operatorname{supp}\left(l_{1}^{\prime}\right)>\operatorname{supp}(g)$ and then $\operatorname{conf}\left(r^{\prime}\right)<\operatorname{conf}(r)$. If $f \subset l_{2}^{\prime}$ then, according to definition 3 , we have $f=\gamma(f) \subset l_{2}^{\prime}=\gamma\left(l_{2}^{\prime}\right)$. We deduce that $\operatorname{supp}(f)>\operatorname{supp}\left(l_{2}^{\prime}\right)$ and we conclude that $\operatorname{conf}(r)>\operatorname{conf}\left(r^{\prime}\right)$. 


\subsection{Non-transitive approximate min-max association RUles}

We can further reduce the number of approximate association rules extracted without losing the ability to deduce all approximate association rules, with support and confidence, by removing transitive min-max association rules.

A min-max association rules $g \rightarrow(f \backslash g)$ with $\gamma(g) \subset f$ is transitive if it exists a frequent closed itemset $f^{\prime}$ such that $\gamma(g) \subset f^{\prime} \subset f$. Let $g^{\prime}$ be the generator of $f^{\prime}$ such that $\gamma(g) \subset g^{\prime} \subseteq f^{\prime} \subset f$. Then, we have the two following approximate min-max association rules: $g \rightarrow\left(f^{\prime} \backslash g\right)$ and $g^{\prime} \rightarrow\left(f \backslash g^{\prime}\right)$. The rule $g \rightarrow(f \backslash g)$ is the transitive composition of the two previous rules; its support is equal to the second rule's support and its confidence is equal to the product of their confidences.

We generalize this characterization to all triplets consisting of a generators $g$, its closure $f$ and a closed superset $f^{\prime}$ of $f$ to define the non-transitive min-max approximate basis, that is the transitive reduction of the min-max approximate basis. Let's denote $l_{1} \lessdot l_{2}$ when an itemset $l_{1}$ is an immediate predecessor of an itemset $l_{2}$, i.e. $\nexists l_{3}$ such that $l_{1} \subset l_{3} \subset l_{2}$. The non-transitive min-max approximate rules are of the form $g \rightarrow(f \backslash g)$ where $f$ is a frequent closed itemset and $g$ a frequent generator such that $\gamma(g)$ is an immediate predecessor of $f$.

Definition 8. (Non-transitive min-max approximate basis) The non-transitive minmax approximate basis is:

$$
\text { MinMaxReduc }=\{r: g \rightarrow(f \backslash g) \mid f \in \text { Closed } \wedge g \in \text { Gen } \wedge \gamma(g) \lessdot f\} .
$$

Remark. This transitive reduction decreases the number of approximate rules extracted, by selecting the most precise rules, i.e. whith highest confidences, since transitive rules have lower confidences than non-transitive rules.

The algorithm presented in figure 6 constructs the set MinMaxReduc of non-transitive approximate min-max rules using frequent closed itemsets and their generators. For each generator $g$, it determines all frequent closed itemsets $f$ that are immediate successors of the closure of $g$ and then, it generates all rules between $g$ and $f$ that have a sufficient confidence.

First, MinMaxReduc is initialized with the empty set (step 1) and sets $F C_{k}$ are successively examined in increasing order of $k$ values (steps 2 to 19). For each $k$ generator $g \in F C_{k}$ (steps 3 to 18), the set $\operatorname{ImSucc}_{g}$ of immediate successors of $g$ closure is initialized with the empty set (step 4 ). The sets $S_{j}$ of frequent closed $j$-supersets of $\gamma(g)$ for $|\gamma(g)|<j \leq \mu$ are constructed (steps 5 to 7 ). Then, sets $S_{j}$ are considered successively in ascending order of $j$ values (steps 8 to 17). For each itemset $f \in S_{j}$ that is not a superset of an immediate successor of $\gamma(g)$ in $\operatorname{ImSucc}_{g}$ (step 10), $f$ is inserted in $\operatorname{ImSucc}_{g}$ (step 11) and the confidence of the rule $r: g \rightarrow(f \backslash g)$ is computed (step 12). If the confidence of $r$ is above minconf, the rule $r$ is inserted in MinMaxReduc (steps 13 and 14). When all the generators of size lower than $\nu-1$ have been considered, the algorithm returns the set MinMaxReduc (step 20).

Example 7. The non-redundant min-max approximate basis extracted from context $\mathcal{D}$ for minsupp $=2 / 6$ and minconf $=2 / 5$ is presented in table VII. It contains 
Input : $\quad$ sets $F C_{k}$, confidence threshold minconf

Output : $\quad$ set MinMaxReduc

1) MinMaxReduc $\leftarrow \varnothing$

2) for $k=1$ to $\nu-1$ do

3) forall $k$-generator $g \in F C_{k}$ do

4) $\quad$ ImSucc $_{g} \leftarrow \varnothing$

5) for $j=\mid$ g.closure $\mid$ to $\mu$ do

6) $\quad S_{j} \leftarrow\{f \in F C$.closure $\mid f \supset$ g.closure $\wedge|f|=j\}$

7) end

8) for $j=\mid$ g.closure $\mid$ to $\mu$ do

9) forall frequent closed itemset $f \in S_{j}$ do

10) $\quad$ if ( $\left.\nexists s \in \operatorname{ImSucc}_{g} \mid s \subset f\right)$ then do

11) insert $f$ in $\operatorname{ImSucc}_{g}$

12) conf $\leftarrow$ f.supp/g.supp

13) $\quad$ if ( conf $\geq \operatorname{minconf})$

14) then insert $\{r: g \rightarrow(f \backslash g)$, conf, f.supp $\}$ in MinMaxReduc

15) end

16) end

17) end

18) end

19) end

20) return MinMaxReduc

Figure 6. Algorithm for generating the non-transitive min-max approximate basis.

only seven rules, that is three rules less than the approximate min-max basis. These three rules are $\mathrm{B} \rightarrow \mathrm{ACE}, \mathrm{C} \rightarrow \mathrm{BE}$ and $\mathrm{E} \rightarrow \mathrm{ABC}$ that have minimal support and confidence measures among the ten rules of the approximate min-max basis.

Table VII. Non-transitive min-max approximate basis extracted from $\mathcal{D}$.

\begin{tabular}{|lllccc|}
\hline Generator & Closure & Closed superset & Approximate rule & Supp & Conf \\
\hline$\{\mathrm{A}\}$ & $\{\mathrm{AC}\}$ & $\{\mathrm{ABCE}\}$ & $\mathrm{A} \rightarrow \mathrm{BCE}$ & $2 / 6$ & $2 / 3$ \\
$\{\mathrm{~B}\}$ & $\{\mathrm{BE}\}$ & $\{\mathrm{BCE}\}$ & $\mathrm{B} \rightarrow \mathrm{CE}$ & $4 / 6$ & $4 / 5$ \\
$\{\mathrm{~B}\}$ & $\{\mathrm{BE}\}$ & $\{\mathrm{ABCE}\}$ & & & \\
$\{\mathrm{C}\}$ & $\{\mathrm{C}\}$ & $\{\mathrm{AC}\}$ & $\mathrm{C} \rightarrow \mathrm{A}$ & $3 / 6$ & $3 / 5$ \\
$\{\mathrm{C}\}$ & $\{\mathrm{C}\}$ & $\{\mathrm{BCE}\}$ & $\mathrm{C} \rightarrow \mathrm{BE}$ & $4 / 6$ & $4 / 5$ \\
$\{\mathrm{C}\}$ & $\{\mathrm{C}\}$ & $\{\mathrm{ABCE}\}$ & & & \\
$\{\mathrm{E}\}$ & $\{\mathrm{BE}\}$ & $\{\mathrm{BCE}\}$ & $\mathrm{E} \rightarrow \mathrm{BC}$ & $4 / 6$ & $4 / 5$ \\
$\{\mathrm{E}\}$ & $\{\mathrm{BE}\}$ & $\{\mathrm{ABCE}\}$ & & & \\
$\{\mathrm{AB}\}$ & $\{\mathrm{ABCE}\}$ & & & & \\
$\{\mathrm{AE}\}$ & $\{\mathrm{ABCE}\}$ & & & & \\
$\{\mathrm{BC}\}$ & $\{\mathrm{BCE}\}$ & $\{\mathrm{ABCE}\}$ & $\mathrm{BC} \rightarrow \mathrm{AE}$ & $2 / 6$ & $2 / 4$ \\
$\{\mathrm{CE}\}$ & $\{\mathrm{BCE}\}$ & $\{\mathrm{ABCE}\}$ & $\mathrm{CE} \rightarrow \mathrm{AB}$ & $2 / 6$ & $2 / 4$ \\
\hline
\end{tabular}


Proposition 5. All approximate association rules, with support and confidence, can be deduced from the non-transitive min-max approximate basis.

First, we show that all approximate min-max association rules can be derived from the non-transitive min-max approximate association rules. Then, from proposition 4 we conclude that all approximate association rules can also be deduced.

Proof. Let $r: g_{1} \rightarrow\left(f_{n} \backslash g_{1}\right)$ be an approximate min-max association rule between a generator $g_{1}$ whose closure is $f_{1}$ and a frequent closed superset $f_{n}$ of $f_{1}$. If $f_{1} \lessdot f_{n}$ then $r$ is non-transitive: $r \in$ MinMaxReduc. If $f_{1} \Varangle f_{n}$ then $r$ is transitive and there is a sequence $f_{1}, f_{2}, \ldots, f_{n}$ of frequent closed itemsets such that $g_{1} \subseteq f_{1} \lessdot f_{2} \lessdot \ldots \lessdot f_{n}$ with $n \geq 3$. Each $f_{i}$ has at least one generator $g_{i}$ such that $\gamma\left(g_{i}\right)=f_{i}$ and since $f_{1} \lessdot f_{2} \lessdot \ldots \lessdot f_{n}$, there is a sequence of rules $r_{i}: g_{i} \rightarrow\left(f_{i+1} \backslash g_{i}\right)$ for $i \in[1, n-1]$ that are non-transitive min-max rules. The antecedent of $r$ is the antecedent $g_{1}$ of the first rule $r_{1}$ of the sequence. The consequent of $r$ is $\left(f_{n} \backslash g_{1}\right)=\left(\left(\left(f_{n} \backslash g_{n-1}\right) \cup g_{n-1}\right) \backslash g_{1}\right)$, i.e. the union of rule $r_{n-1}$ 's antecedent and consequent minus rule $r_{1}$ 's antecedent. We now show that support and confidence of $r$ can be deduced of those of rules $r_{i}$. We have $\operatorname{supp}(r)=\operatorname{supp}\left(g_{1} \cup\left(f_{n} \backslash g_{1}\right)\right)=\operatorname{supp}\left(f_{n}\right)=\operatorname{supp}\left(g_{n-1} \cup\left(f_{n} \backslash g_{n-1}\right)\right)=\operatorname{supp}\left(r_{n-1}\right)$. The support of $r$ is equal to the support of the last rule $r_{n-1}$ of the sequence. We also have: $\operatorname{conf}(r)=\operatorname{supp}\left(f_{n}\right) / \operatorname{supp}\left(g_{1}\right)=\operatorname{supp}\left(f_{n}\right) / \operatorname{supp}\left(g_{n-1}\right) \times \operatorname{supp}\left(g_{n-1}\right) / \operatorname{supp}\left(g_{1}\right)$ $=\operatorname{supp}\left(f_{n}\right) / \operatorname{supp}\left(g_{n-1}\right) \times \operatorname{supp}\left(f_{n-1}\right) / \operatorname{supp}\left(g_{n-2}\right) \times \ldots \times \operatorname{supp}\left(f_{2}\right) / \operatorname{supp}\left(g_{1}\right)=$ $\operatorname{conf}\left(r_{n-1}\right) \times \operatorname{conf}\left(r_{n-2}\right) \times \ldots \times \operatorname{conf}\left(r_{1}\right)$. The confidence of $r$ is equal to the product of the confidences of the rules $r_{i}$ for $i=1$ to $n-1$.

\section{Deriving association rules from the min-max bases}

We introduce in this section simple techniques and algorithms to reconstruct all exact association rules, all approximate association rules and all transitive approximate min-max association rules from the min-max bases.

\subsection{Deriving exact association RUles}

The graph-oriented representation of the exact and the exact min-max association rules extracted from context $\mathcal{D}$ for minsupp $=2 / 6$ and minconf $=2 / 5$ are given in figure 7 and 8 respectively.

Each vertex $v_{l}$ represents a frequent itemset $l$ that is a subset of the maximal frequent itemset $\{\mathrm{ABCE}\}$. Each edge between two vertices $v_{a}$ and $v_{c}$ represents the exact association rule $a \Rightarrow c \backslash a$. A closed interval is a sub-graph containing all vertices representing itemsets of the intervals $\left[g_{i}, f\right]$ where each $g_{i}$ is a generator of the frequent closed itemset $f$. Since all itemsets in a closed interval have the same support, all rules in this interval also have the same support.

In the graph representation, deriving all exact rules means adding all possible edges between two vertices of the same closed interval. Each edge in figure 8 between two vertices $v_{g}$ and $v_{f}$ represents a rule between a generator $g$ and its closure $f$. Then, 


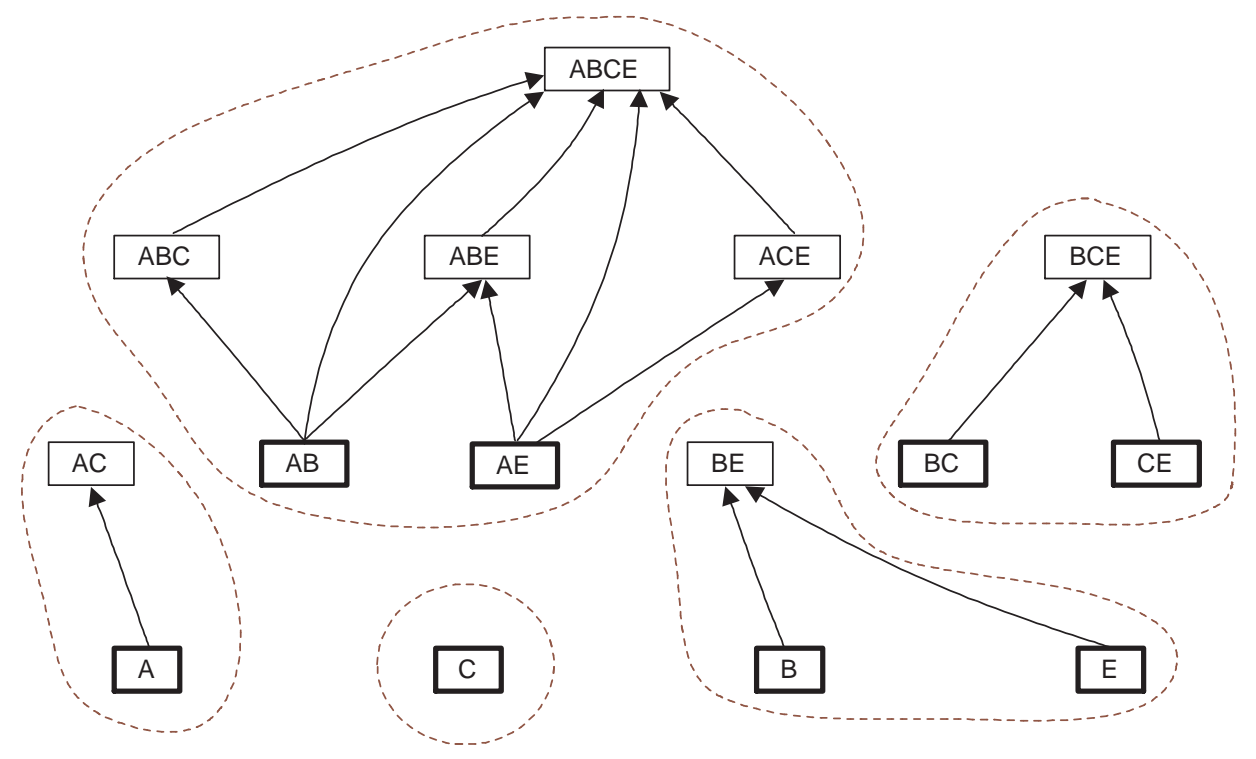

$\square$ generator itemset

closed interval

Figure \%. Exact association rules extracted from $\mathcal{D}$.

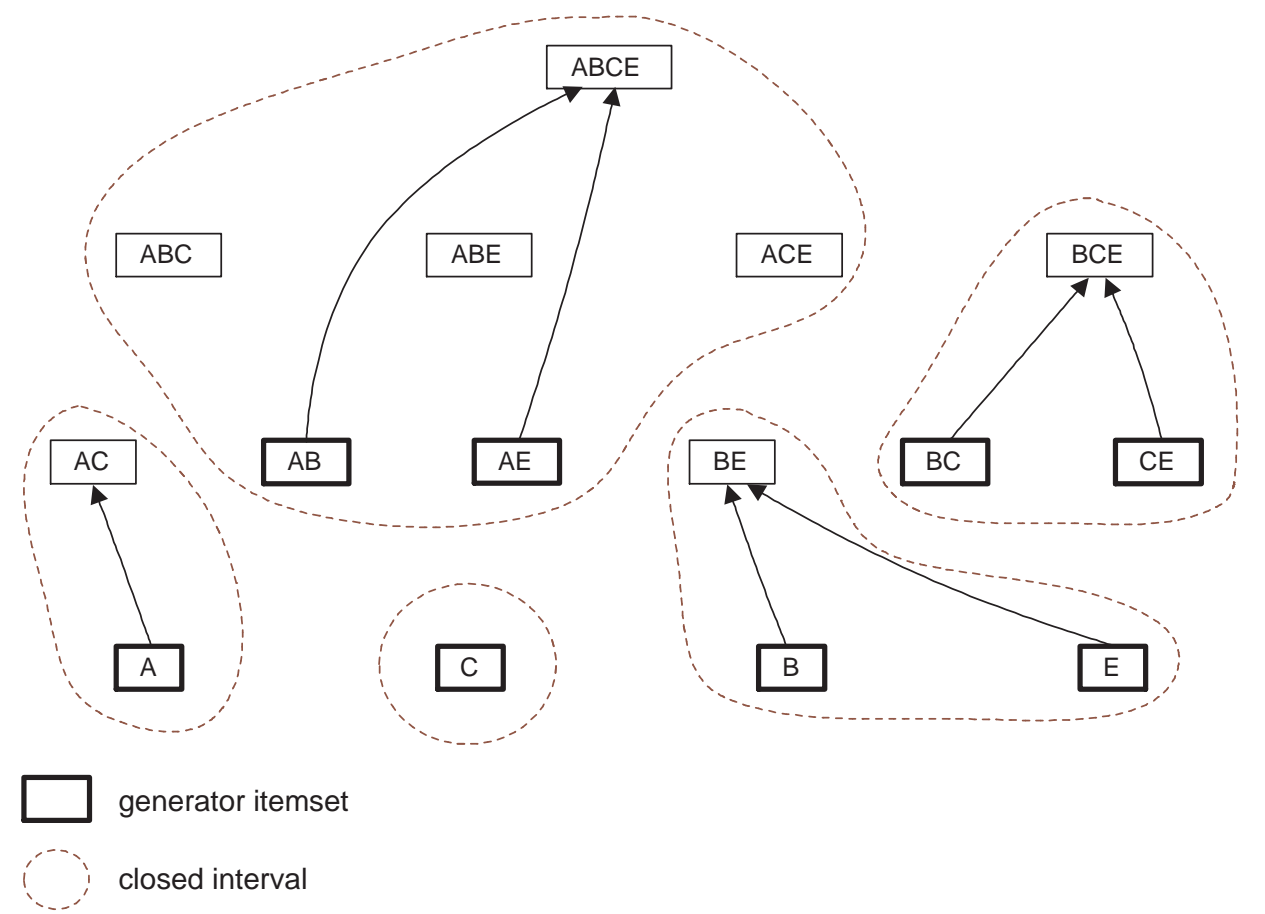

Figure 8. Exact min-max association rules extracted from $\mathcal{D}$.

we add all edges between two vertices, one representing a superset of $g$ and the other a subset of $f$. 
The algorithm receives the set MinMaxExact of exact min-max rules as input and it returns the set AllExact containing all exact association rules. Its pseudo-code is presented in figure 9. It considers all exact min-max rules $r_{1}: a_{1} \Rightarrow c_{1}$ with $\left|c_{1}\right|>1$ (steps 2 to 8 ). For all subset $c_{2}$ of $c_{1}$ (steps 3 to 7 ), it generates all rules with the form $r_{2}: a_{1} \Rightarrow c_{2}$ and $r_{3}: a_{1} \cup c_{2} \Rightarrow c_{1} \backslash c_{2}$ (steps 4 and 6 ). These rules have the same support as $r_{1}$. Since rule $r_{3}$ can be generated several times, the algorithm first tests if it has not already been inserted in AllExact (step 5).

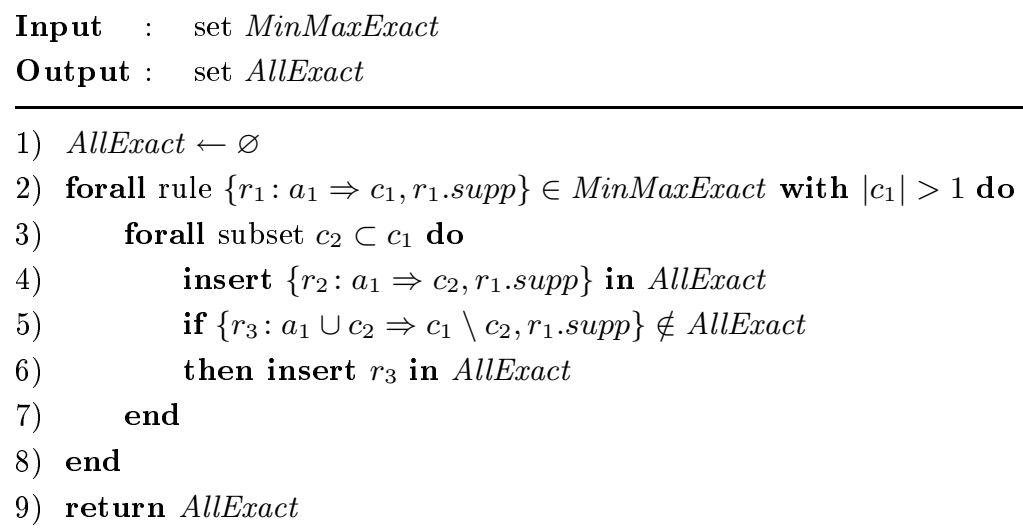

Figure 9. Algorithm for reconstructing all exact association rules.

Example 8. Consider rule $\mathrm{AB} \Rightarrow \mathrm{CE}$ represented in figure 4 by the edge between vertices $\{A B\}$ and $\{A B C E\}$. From this rule we deduce rules $A B \Rightarrow C, A B \Rightarrow E$, $\mathrm{ABC} \Rightarrow \mathrm{E}$ and $\mathrm{ABE} \Rightarrow \mathrm{C}$ and from rule $\mathrm{AE} \Rightarrow \mathrm{BC}$, we deduce rules $\mathrm{AE} \Rightarrow \mathrm{B}$, $\mathrm{AE} \Rightarrow \mathrm{C}, \mathrm{ABE} \Rightarrow \mathrm{C}$ and $\mathrm{ACE} \Rightarrow \mathrm{B}$. All these rules have the same support.

Remark. For constructing all exact rules using sets $F C_{k}$ of generators and frequent closed itemsets, we consider each generator $g$ and its closure $f$. We generate all rules $r: g \Rightarrow l \backslash g$ and $r: l \Rightarrow f \backslash l$ for $l \in[g, f[$. For instance, from the generator $\{\mathrm{AB}\}$ and its closure $\{\mathrm{ABCE}\}$, we generate rules $\mathrm{AB} \Rightarrow \mathrm{CE}, \mathrm{AB} \Rightarrow \mathrm{C}, \mathrm{AB} \Rightarrow \mathrm{E}, \mathrm{ABC} \Rightarrow \mathrm{E}$ and $\mathrm{ABE} \Rightarrow \mathrm{C}$. Their support is equal to the support of $g$ and $f$, i.e. the support of $\{\mathrm{AB}\}$ and $\{\mathrm{ABCE}\}$.

\subsection{Deriving APPROXIMATE ASSOCIATION RULES}

Figures 10 and 11 depict the graph-oriented representations of the approximate and the approximate min-max association rules extracted from context $\mathcal{D}$ for minsupp $=2 / 6$ and $\operatorname{minconf}=2 / 5$. Each edge between two vertices $v_{a}$ and $v_{c}$ represents the approximate rule $a \rightarrow c \backslash a$.

In figure 11, each edge between two vertices $v_{g}$ and $v_{f}$ represents the min-max approximate rule $g \rightarrow f \backslash g$ where $g$ is a generator and $f$ a frequent closed superset of $g$. That is to say an edge between a minimal vertex of a closed interval and the maximal vertex of another closed interval above the first one. For instance, the edge between vertices containing $\{\mathrm{A}\}$ and $\{\mathrm{ABCE}\}$ represents the rule $\mathrm{A} \rightarrow \mathrm{BCE}$. 


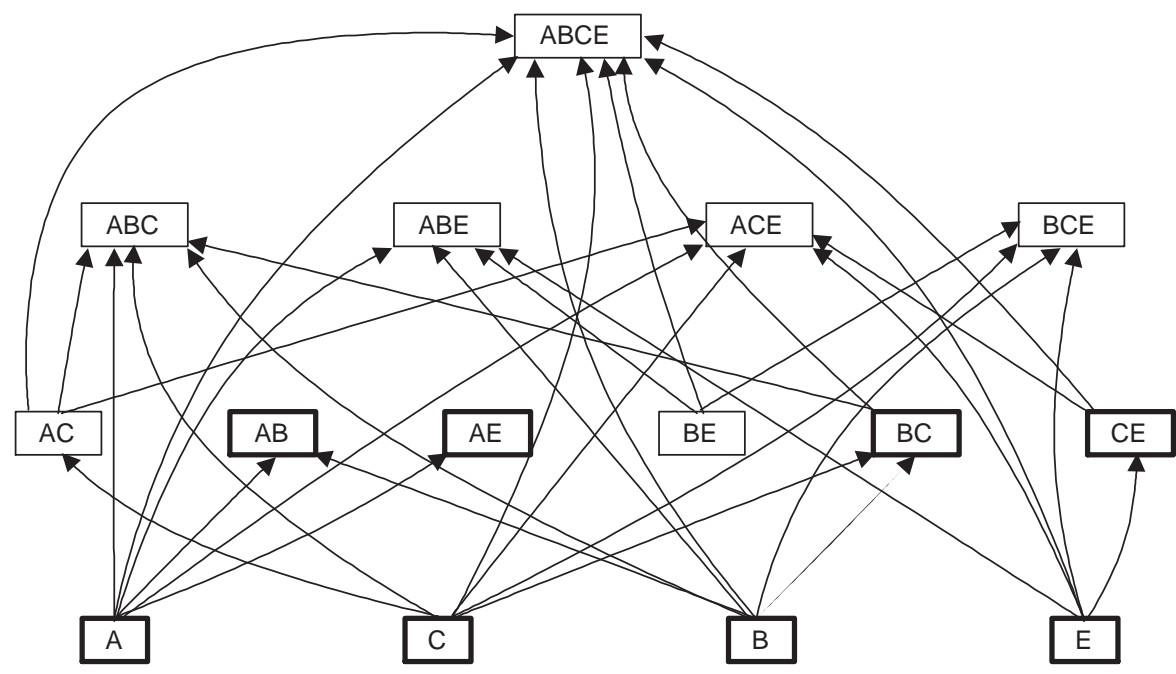

generator itemset

Figure 10. Approximate association rules extracted from $\mathcal{D}$.

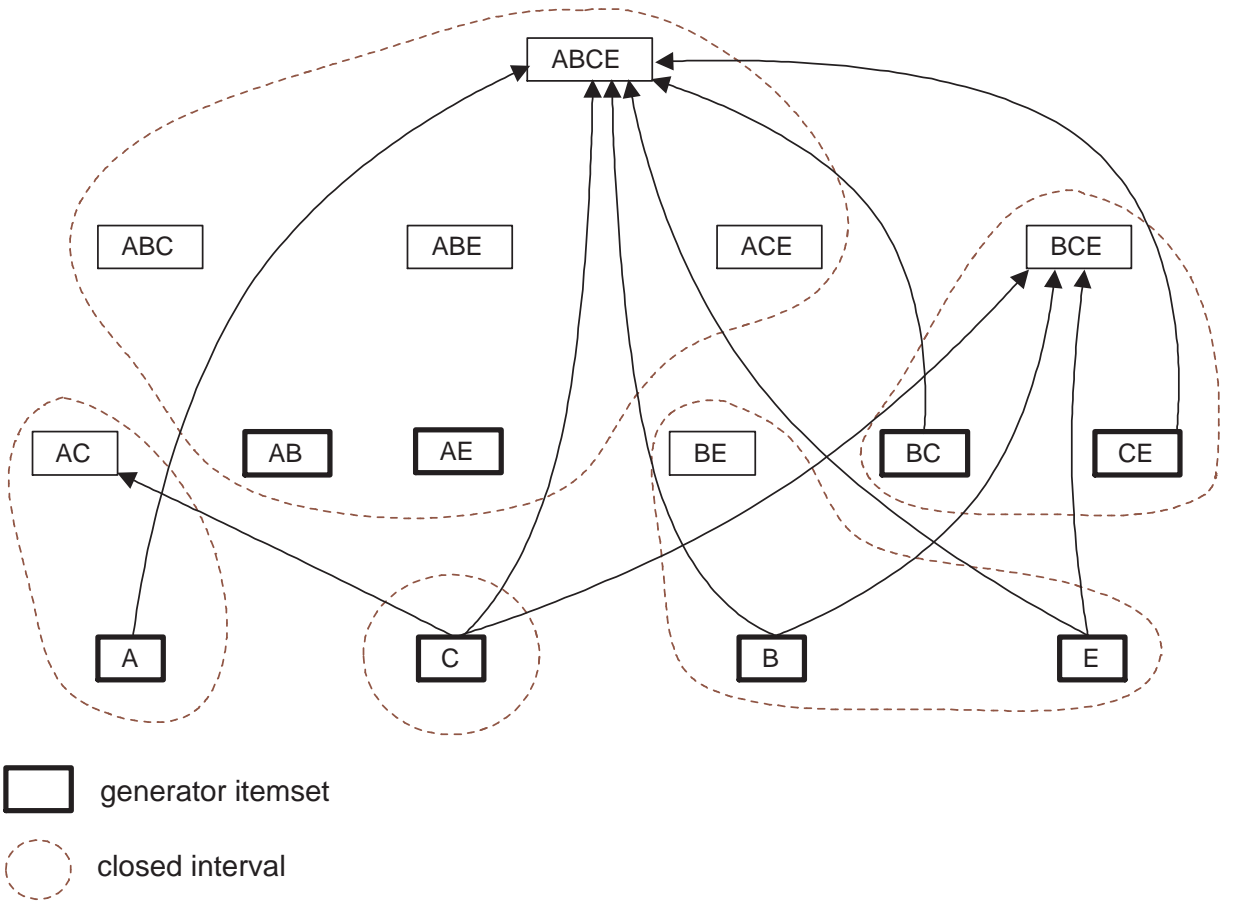

Figure 11. Approximate min-max association rules extracted from $\mathcal{D}$.

To derive all approximate rules, when there is an edge between two vertices of two closed intervals we create all possible edges between each vertex of the first interval and each vertex of the second interval. All these rules have the same support and confidence. In figure 11 for instance, we add all edges between vertices of the closed 
interval $\{\{\mathrm{A}\},\{\mathrm{AC}\}\}$ and the closed interval $\{\{\mathrm{AB}\},\{\mathrm{AE}\},\{\mathrm{ABC}\},\{\mathrm{ABE}\},\{\mathrm{ACE}\}$, $\{\mathrm{ABCE}\}$. These rules have the same support and confidence as rule $\mathrm{A} \rightarrow \mathrm{BCE}$.

A simple and efficient method to derive all approximate rules is to proceed in two phases. First, we generate all rules with the form $g_{1} \rightarrow l_{i} \backslash g_{1}$ between a generator $g_{1}$ and all its frequent supersets $l_{i} \in\left[g_{i}, f_{i}\right]$ where $g_{i}$ is a generator of $f_{i}$ and $g_{1} \subset g_{i}$. Second, we "extend" these rules by replacing their antecedent by all itemsets $l_{1} \in$ $\left[g_{1}, f_{1}\right]$ where $f_{1}$ is the closure of $g_{1}$.

The input of the algorithm are the sets MinMaxApprox and MinMaxExact of approximate and exact min-max rules. Its result is the set AllApprox containing all approximate rules. Its pseudo-code is presented in figure 12 .

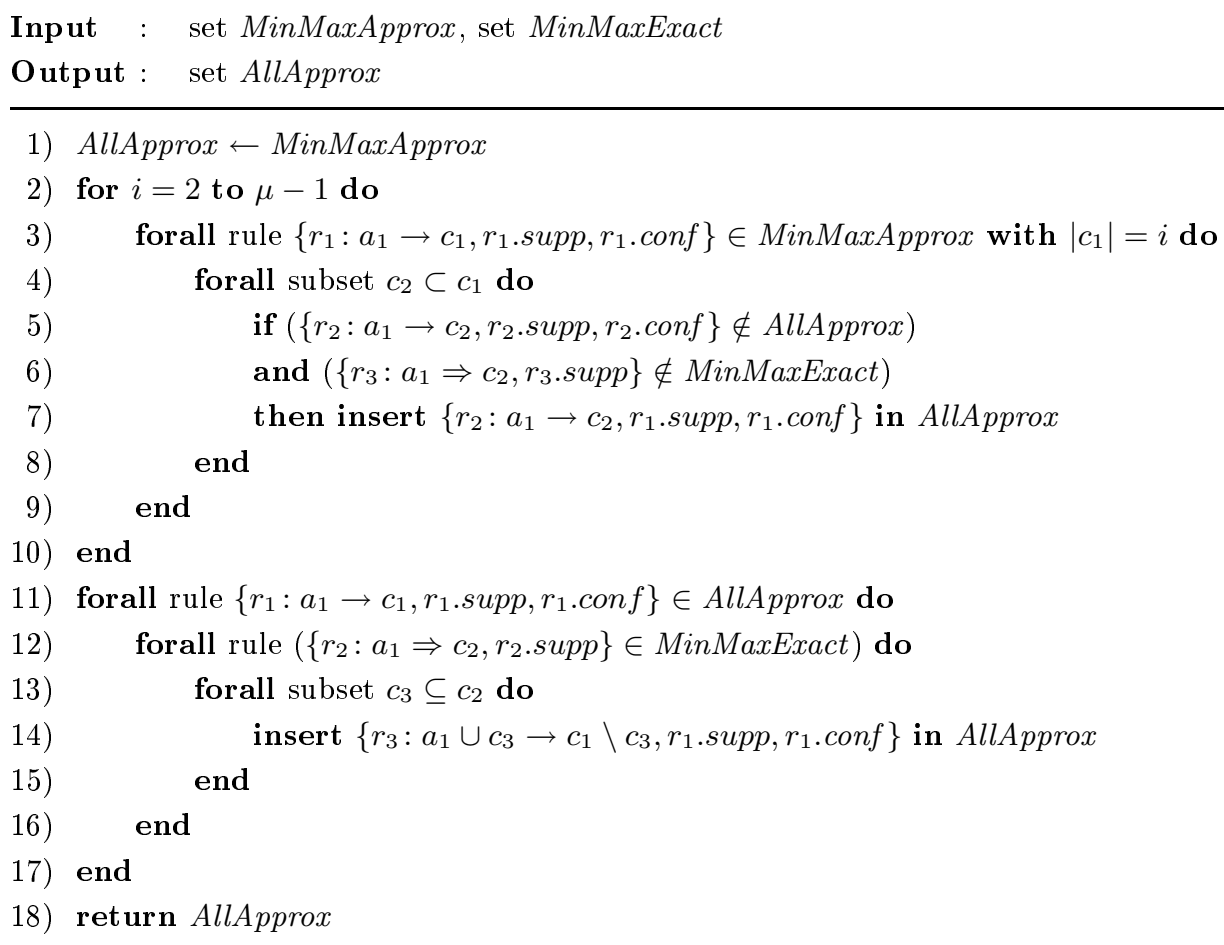

Figure 12. Algorithm for reconstructing approximate min-max association rules.

In the first phase (steps 2 to 10), it considers min-max approximate rules $a_{1} \rightarrow c_{1}$ with $\left|c_{1}\right|>1$ in increasing order of their consequent's size (steps 3 to 9 ). For each min-max rule $a_{1} \rightarrow c_{1}$, all rules with the form $a_{1} \rightarrow c_{2}$ with $c_{2} \subset c_{1}$ are generated if they were not previously generated and there is no exact rule $a_{1} \Rightarrow c_{2}$ (steps 4 to 8 ). All these rules have the same support and confidence. In the second phase(steps 11 to 17), it considers all approximate rules $a_{1} \rightarrow c_{1}$ and for each min-max exact rule $a_{1} \Rightarrow c_{2}$ (steps 12 to 16 ), it generates all rules with the form $a_{1} \cup c_{3} \rightarrow c_{1} \backslash c_{3}$ for all subset $c_{3}$ of $c_{2}$ (steps 13 to 15 ).

Example 9. Considering rule $\mathrm{A} \rightarrow \mathrm{BCE}$ in figure 11, we deduce rules $\mathrm{A} \rightarrow \mathrm{B}, \mathrm{A} \rightarrow \mathrm{E}$, $\mathrm{A} \rightarrow \mathrm{BC}, \mathrm{A} \rightarrow \mathrm{BE}, \mathrm{A} \rightarrow \mathrm{CE}$. Rule $\mathrm{A} \rightarrow \mathrm{C}$ is not generated since $\mathrm{A} \Rightarrow \mathrm{C}$ is an exact rule, i.e. $\{\mathrm{A}\}$ and $\{\mathrm{AC}\}$ belong to the same closed interval. Then, since we have 
$\mathrm{A} \Rightarrow \mathrm{C}$, extending all rules with $\mathrm{A}$ as antecedent we obtain rules $\mathrm{AC} \rightarrow \mathrm{B}, \mathrm{AC} \rightarrow \mathrm{E}$, $\mathrm{AC} \rightarrow \mathrm{BE}$.

In order to generate all approximate rules using sets $F C_{k}$ of generators and frequent closed itemsets, we consider each couple of intervals $\left\{\left[g_{1}, f_{1}\right],\left[g_{2}, f_{2}\right]\right\}$ with $\gamma\left(g_{1}\right)=f_{1}$ and $\gamma\left(g_{2}\right)=f_{2}$ such that $g_{1} \subset g_{2}$. We generate all rules $r: l_{1} \rightarrow l_{2} \backslash l_{1}$ for $l_{1} \in\left[g_{1}, f_{1}\right]$ and $l_{2} \in\left[g_{2}, f_{2}\right]$. The support of these rules is $\operatorname{supp}\left(f_{2}\right)$ and their confidence is $\operatorname{supp}\left(f_{2}\right) / \operatorname{supp}\left(f_{1}\right)$. For instance, from the generator $\{\mathrm{B}\}$ and its closure $\{\mathrm{BE}\}$ and the generator $\{\mathrm{BC}\}$ and its closure $\{\mathrm{BCE}\}$, we generate the rules $\mathrm{B} \rightarrow \mathrm{C}, \mathrm{B} \rightarrow \mathrm{CE}$ and $\mathrm{BE} \rightarrow \mathrm{C}$.

\subsection{DeRIVING TRANSitive APPROXimate Min-MAX ASSOCIATION RUles}

The graph-oriented representation of the non-transitive approximate min-max association rules extracted from context $\mathcal{D}$ for minsupp $=2 / 6$ and minconf $=2 / 5$ is given in figure 13 .

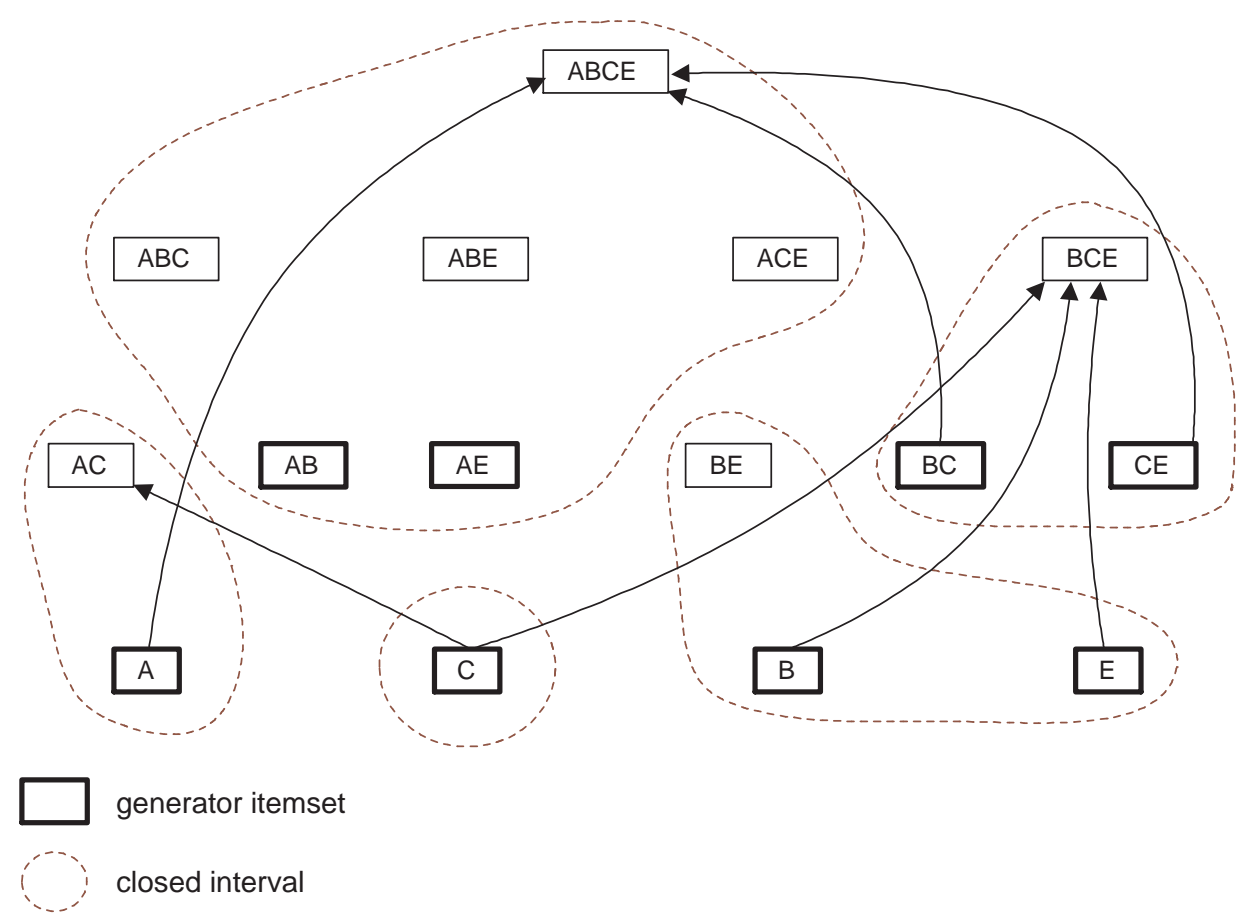

Figure 13. Non-transitive approximate min-max association rules extracted from $\mathcal{D}$.

Each edge between two vertices $v_{g}$ and $v_{f}$ represents the non-transitive approximate rule $g \rightarrow f \backslash g$ where $g$ is a generator and $f$ a frequent closed immediate successor of the closure of $g$. That is an edge between a minimal vertex of a closed interval and the maximal vertex of an immediately above closed interval.

An edge in figure 11 represents a transitive rule if it is an edge between a minimal vertex of a closed interval and the maximal vertex of another closed interval that is not immediately above the first one: There is a closed interval "intermediate" between these two intervals. For instance, the rule $\mathrm{C} \rightarrow$ ABE between the closed 
intervals $\{\{\mathrm{C}\}\}$ and $\{\{\mathrm{AB}\},\{\mathrm{AE}\},\{\mathrm{ABC}\},\{\mathrm{ABE}\},\{\mathrm{ACE}\},\{\mathrm{ABCE}\}\}$ is transitive since we have rules $\mathrm{C} \rightarrow \mathrm{A}$ and $\mathrm{A} \rightarrow \mathrm{BCE}$ and the closed interval $\{\{\mathrm{A}\},\{\mathrm{AC}\}\}$ is intermediate, i.e., $\{\mathrm{C}\} \subset\{\mathrm{AC}\} \subset\{\mathrm{ABCE}\}$. The confidence of $\mathrm{C} \rightarrow \mathrm{ABE}$ is equal to the product of rules $\mathrm{C} \rightarrow \mathrm{A}$ and $\mathrm{A} \rightarrow \mathrm{BCE}$ confidences.

In order to derive all transitive rules, we first add all rules that are compositions of two non-transitive rules, we then derive from them rules that are compositions of three non-transitive rules and so on until no new rule can be derived. The three transitive min-max rules reconstructed are $\mathrm{C} \rightarrow \mathrm{ABE}, \mathrm{B} \rightarrow \mathrm{ACE}$ and $\mathrm{E} \rightarrow \mathrm{ABC}$. They are all compositions of two non-transitive rules, that have the form $g_{i} \rightarrow f_{j} \backslash g_{i}$ with $g_{i} \subseteq \gamma\left(g_{i}\right)=f_{i} \lessdot f_{j}$, represented in figure 7 .

The algorithm presented in figure 14 generates the set MinMaxApprox of approximate min-max rules using the set MinMaxReduc of non-transitive approximate min-max rules and the minconf threshold as its input.

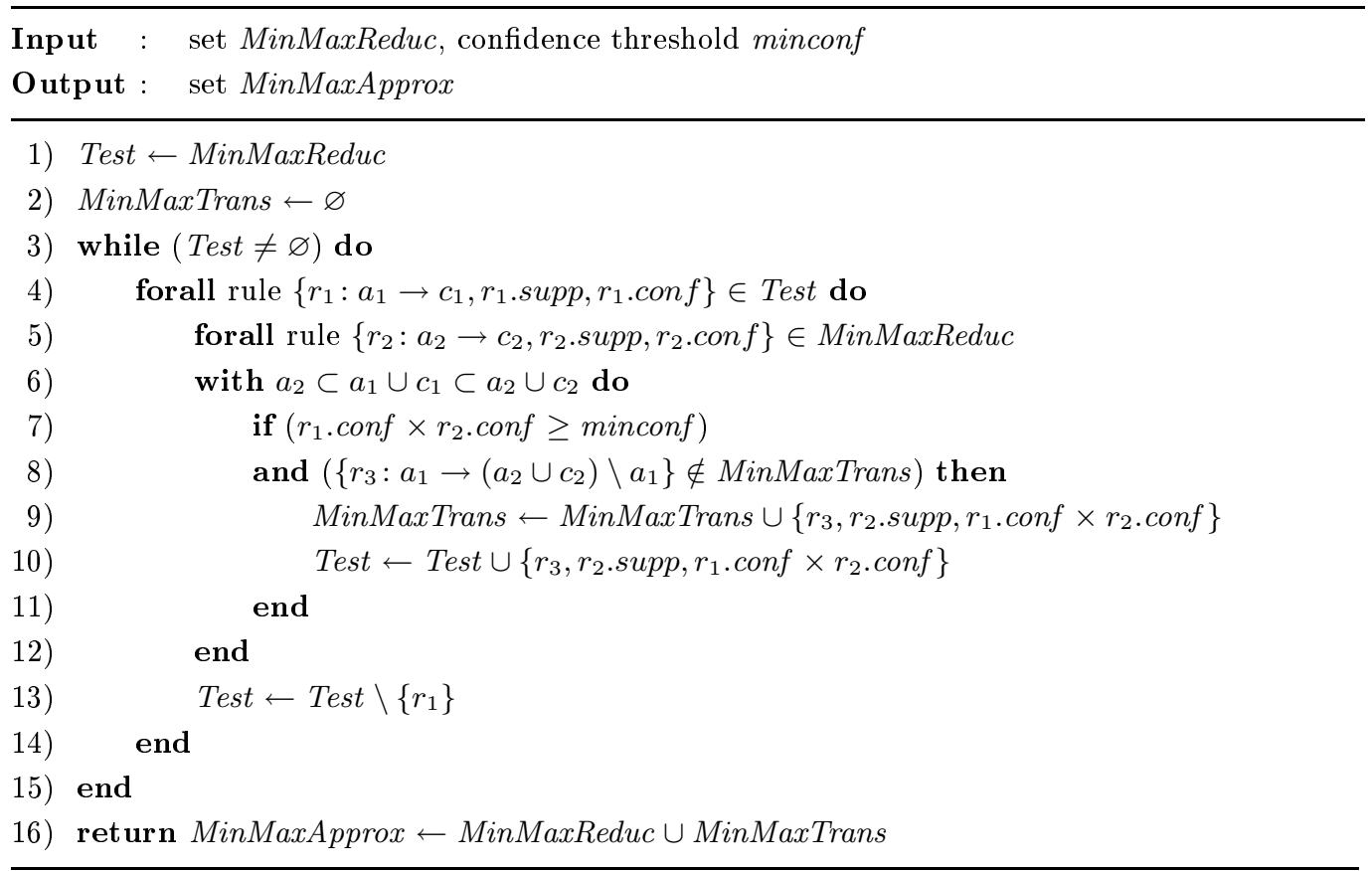

Figure 14. Algorithm for reconstructing transitive approximate min-max association rules.

The approach is incremental: We iteratively add new transitive min-max rules until no new rule has been created (steps 3 to 15). During each iteration, the Test set contains all rules examined to generate new transitive rules and the algorithm stops when Test is empty. This set is initialized with all non-transitive rules (step 1) and all rules $r_{1}$ it contains, that have the form $g_{i} \rightarrow f_{j} \backslash g_{i}$, are successively examined (steps 4 to 14). For each non-transitive rule $r_{2}$ in MinMaxReduc with the form $g_{j} \rightarrow f_{m} \backslash g_{j}$ such that $g_{j} \subset f_{j} \subset f_{m}$ (steps 5 and 6 to 12), the transitive rule $r_{3}$ with the form $g_{i} \rightarrow f_{m} \backslash g_{i}$ is generated in MinMaxTrans and Test (steps 9 and 10) if its confidence is sufficient and it is not already present in MinMaxTrans (steps 7 
and 8). Then, rule $r_{1}$ is removed from Test (step 13) since it is not needed anymore: Only transitive rules generates from $r_{1}$ will be examined in the following iterations.

Example 10. The transitive rule $\mathrm{B} \rightarrow \mathrm{ACE}$ is derived from rules $\mathrm{B} \rightarrow \mathrm{CE}$ and $\mathrm{BC} \rightarrow \mathrm{AE}$ whose antecedent $\{\mathrm{BC}\}$ is a subset of $\{\mathrm{B}\} \cup\{\mathrm{CE}\}=\{\mathrm{BCE}\}$, and $\{\mathrm{BCE}\}$ is itself a subset of $\{\mathrm{BC}\} \cup\{\mathrm{AE}\}=\{\mathrm{ABCE}\}$. The rule $\mathrm{E} \rightarrow \mathrm{ABC}$ is derived from $\mathrm{E} \rightarrow \mathrm{BC}$ and $\mathrm{CE} \rightarrow \mathrm{AB}$. The rule $\mathrm{C} \rightarrow \mathrm{ABE}$ can be derived from rules $\mathrm{C} \rightarrow \mathrm{A}$ and $\mathrm{A} \rightarrow \mathrm{BCE}$, or from rules $\mathrm{C} \rightarrow \mathrm{BE}$ and $\mathrm{BC} \rightarrow \mathrm{AE}$ or $\mathrm{CE} \rightarrow \mathrm{AB}$.

\section{Experimental results}

We used the four following datasets during these experiments: T10I4D100K ${ }^{5}$ is a synthetic dataset built according to sales data properties. It contains 100,000 objects with an average object size of 10 items and an average size of potential maximal frequent itemsets of 4 items. The Mushrooms dataset describes 23 characteristics (attributes) of 8,416 mushrooms (objects): Each object is related to 23 items and we have 127 items on the whole. The C20D10K and C73D10K (Hettich and Bay, 1999) datasets are samples of the 1990 census in Kansas, each containing 10,000 objects corresponding to the first 10,000 listed people. Each object is described by 20 attributes (20 items by objects and 386 items on the whole) in C20D10K and 73 attributes (73 items by objects and 2,178 items on the whole) in C73D10K.

Running times of the generation of all association rules and of the min-max bases are not shown since they are insignificant compared to execution times of the itemset extraction. Indeed, no dataset scan is required for this phase and all computations take place in main memory. As a data-point, the largest running time obtained was 46.27 seconds for the generation of the 2,053,936 approximate association rules for $\mathrm{C} 73 \mathrm{D} 10 \mathrm{~K}$ on a Pentium II at $333 \mathrm{MHz}$ with $256 \mathrm{MB}$ of main memory.

Number of exact association rules extracted. The total number of exact association rules and the number of min-max exact association rules are presented in table VIII. No exact association rule is extracted from T10I4D100K since, for this minsupp value, all frequent itemsets are frequent closed itemsets. Thus, they are themselves their own unique generator and consequently, there is no exact association rule $l_{1} \Rightarrow\left(l_{2} \backslash l_{1}\right)$ between two frequent itemsets $l_{1} \subset l_{2}$ having identical closures $\gamma\left(l_{1}\right)=\gamma\left(l_{2}\right)$. The three other datasets are made up of correlated data, and the total number of exact rules is important, making it difficult to discover interesting information. For these datasets, the min-max exact basis reduces the number of rules by a factor varying from 13 to 50 . Since there is no information loss, it brings a complete summary of relevant information that is easier to exploit for the analyst.

Number of approximate association rules extracted. The total number of approximate association rules and the number of approximate and non-transitive approximate min-max rules are presented in table IX. The number of approximate

\footnotetext{
${ }^{5}$ http $: / / w w w . a l m a d e n . i b m . c o m / c s / q u e s t / s y n d a t a . h t m l$
} 
Table VIII. Number of exact association rules extracted.

\begin{tabular}{|cccc|}
\hline Dataset & minsupp & Exact rules & Min-max basis \\
\hline T10I4D100K & $0.5 \%$ & 0 & 0 \\
Mushrooms & $30 \%$ & 7,476 & 543 \\
C20D10K & $50 \%$ & 2,277 & 457 \\
C73D10K & $90 \%$ & 52,035 & 1,369 \\
\hline
\end{tabular}

rules is very significant for the four datasets, up to more than 2,000,000. Reducing this number is thus essential in order to make it usable by the analyst. For T10I4D100K, all frequent itemsets are both closed and their own generators and the approximate min-max basis is identical to the set of all rules. The non-transitive basis represents a reduction by a factor of 5 approximately of the number of rules. For the three other datasets, the total number of approximate rules is much more important than for the synthetic dataset since they contain dense and correlated data: The number of frequent itemsets is much more important and thus, it is the same for the number of approximate rules. However, the fraction of frequent itemsets that are closed is small and the bases reduce considerably the number of rules, by a factor of varying from 10 to 50 for the approximate min-max basis and, from 40 to 500 for the non-transitive basis.

Table IX. Number of approximate association rules extracted.

\begin{tabular}{|ccccc|}
\hline $\begin{array}{c}\text { Dataset } \\
\text { (minsupp) }\end{array}$ & minconf & $\begin{array}{c}\text { Approximate } \\
\text { rules }\end{array}$ & $\begin{array}{c}\text { Approximate } \\
\text { min-max basis }\end{array}$ & $\begin{array}{c}\text { Non-transitive } \\
\text { min-max basis }\end{array}$ \\
\hline $\begin{array}{c}\text { T10I4D100K } \\
(0.5 \%)\end{array}$ & $70 \%$ & 20,419 & 20,419 & 4,004 \\
\hline MusHROOMS & $70 \%$ & 22,952 & 22,952 & 4,519 \\
$(30 \%)$ & $30 \%$ & 37,671 & 2,961 & 1,221 \\
\hline C20D10K & $70 \%$ & 71,412 & 6,571 & 1,578 \\
$(50 \%)$ & $30 \%$ & 89,601 & 10,116 & 1,957 \\
\hline C73D10K & $90 \%$ & 116,791 & 13,634 & 1,957 \\
$(90 \%)$ & $80 \%$ & $2,053,896$ & 43,171 & 5,718 \\
\hline
\end{tabular}

Examining rules generated in the min-max approximate basis and its transitive reduction for the Mushrooms dataset, we verified that rule 4 of example 1 in section 1 is the only one generated among the nine rules. Indeed, the itemsets ffree gills\} and \{free gills, edible, partial veil, white veil\} are frequent closed itemsets and the first is an immediate predecessor of the second. Moreover, they are the only frequent closed itemsets in the interval [ $\varnothing,\{$ free gills, edible, partial veil, white veil $\}]$ and the frequent closed itemset \{free gills\} is itself its own unique generator. Thus, rule 4 is the only min-max approximate rule among the nine rules and is nontransitive. 


\section{Conclusion}

The problem of association rules relevance occurs for most operational datasets. This problem is related to the huge number of rules generated and the presence of many redundancies. The approach proposed in this paper consists in generating bases for association rules that minimize as much as possible the number of extracted rules while bringing the same information to the end-user. Using a semantic based on the Galois connection, we first characterized min-max association rules as the nonredundant rules with minimal antecedent and maximal consequent. Each min-max rule summarizes several other rules, suggesting that these rules are the most relevant from the analyst's point of view. From this characterization, we defined the min-max basis for exact association rules, the min-max basis for approximate association rules and its transitive reduction - which we believe is more useful for the analyst as it retains only the most precise rules. The union of the former and one of the latter of these bases constitutes a min-max basis for association rules that is a generating set for all association rules, their supports and their confidences.

We presented algorithms for generating these bases from the frequent closed itemsets and their generators, such as extracted by the CLOSE and A-Close algorithms. When all frequent itemsets have been mined, the $\mathrm{CLOSE}^{+}$algorithm identifies frequent closed itemsets and their generators among frequent itemsets. We also introduced simple methods and algorithms to derive all exact rules, all approximate rules and all transitive approximate min-max rules from the bases. None of these algorithms requires accessing the dataset and their execution times are thus insignificant compared to the running times of the frequent itemsets, or the frequent closed itemsets, extraction.

Experimental results conducted on both synthetic and operational datasets show that the extraction of these bases considerably reduces the number of rules, particularly in the case of dense or correlated data. The result is easier to browse and since redundant - and often misleading - rules are suppressed, its usefulness is improved. Moreover, all of the data-space is characterized by the min-max rules and this approach does not suffer from poorly characterized or uncharacterized sub-spaces of the data-space, an important weakness of many reduction methods. Another interesting feature of this approach is the possibility to construct a graph-oriented representation of the min-max bases that is easily understandable for the end-user. It provides a natural, simple and clear graphical representation of association rules covering all the data-space and from which the deduction of all other rules is direct. An interesting perspective of future work is the definition of an inference system for association rules equivalent to the Armstrong axioms for implications. As pointed out in section 1.1, up to now no complete and sound inference system that takes supports and confidences into consideration has been proposed. Another attractive perspective of future work is the introduction of the min-max bases in the data analysis and the Formal Concept Analysis domains. Indeed, the min-max association rule definition is valid within the global and partial implication rule frameworks. Hence, the definitions of the min-max bases for exact and approximate association rules are also valid for global and partial implication rules respectively. Since these bases represent no information loss and are constituted of the most relevant rules 
from the analyst's point of view, we believe that studying their impact in these domains is also an interesting perpective.

\section{References}

Agrawal R., Imielinski T. and Swami A. Mining association rules between sets of items in large databases. Proceedings of the SIGMOD conference, pp 207-216, May 1993.

Agrawal R. and Srikant R. Fast algorithms for mining association rules in large databases. Proceedings of the VLDB conference, pp 478-499, September 1994.

Armstrong W. W. Dependency structures of data base relationships. Proceedings of the IFIP congress, pp 580-583, August 1974.

Baralis E. and Psaila G. Designing templates for mining association rules. Journal of Intelligent Information Systems, 9(1):7-32, L. Kerschberg, Z. Ras and M. Zemankova editors, Kluwer Academic Publishers, August 1997.

Bastide Y., Taouil Y., Pasquier N., Stumme G. and Lakhal L. Mining frequent patterns with counting inference. SIGKDD Explorations, 2(2):66-75, U. Fayyad, S. Sarawagi and P. Bradley editors, ACM Computer Press, December 2000.

Bayardo R. J. Efficiently mining long patterns from databases. Proceedings of the SIGMOD conference, pp 85-93, June 1998.

Bayardo R. J. and R. Agrawal. Mining the most interesting rules. Proceedings of the KDD conference, pp 145-154, August 1999.

Bayardo R. J., Agrawal R. and Gunopulos D.. Constraint-based rule mining in large, dense databases. Data Mining and Knowledge Discovery, 4(2/3):217-240, S. Chaudhuri editor, Kluwer Academic Publishers, July 2000.

Beeri C. and Bernstein P. A. Computational problems related to the design of normal form relational schemas. Transactions on Database Systems, 4(1):30-59, March 1979.

Blake C. L. and Merz C. J. UCI Machine Learning databases Repository. University of California, Irvine, Department of Information and Computer Science, 1998. http://www.ics.uci.edu/ mlearn/MLRepository.html.

Brin S., Motwani R., Ullman J. D. and Tsur S. Dynamic itemset counting and implication rules for market basket data. Proceedings of the SIGMOD conference, pp 255-264, May 1997.

Brin S., Motwani R and Silverstein C. Beyond market baskets: Generalizing association rules to correlation. Proceedings of the SIGMOD conference, pp 265-276, May 1997.

Duquenne V. and Guigues J.-L. Famille minimale d'implications informatives résultant d'un tableau de données binaires. Mathématiques et Sciences Humaines, 24(95):5-18, 1986.

Ganter B. and Wille R. Formal Concept Analysis: Mathematical foundations. Springer-Verlag, 1999.

Han J. and Fu Y. Mining multiple-level association rules in large databases. Transactions on Knowledge and Data Engineering, 11(5):798-804, P.-S. Yu editor, IEEE Computer Science, September/October 1999.

Hettich S. and Bay S. D. UCI Knowledge Discovery in Databases Archive. University of California, Irvine, Department of Information and Computer Science, 1999. http://kdd.ics.uci.edu.

Klemettinen M., Mannila H., Ronkainen P., Toivonen H. and Verkamo A. I. Finding interesting rules from large sets of discovered association rules. Proceedings of the CIKM conference, $\mathrm{pp}$ 401-407, November 1994.

Lin D. and Kedem Z. M. Pincer-Search: A new algorithm for discovering the maximum frequent set. Proceedings of the EDBT conference, pp 105-119, March 1998.

Liu B., Hsu W. and Ma Y. Pruning and summarizing the discovered association rules. Proceedings of the KDD conference, pp 125-134, August 1999.

Luxenburger M. Implications partielles dans un contexte. Mathématiques, Informatique et Sciences Humaines, 29(113):35-55, 1991.

Maier D. Minimum covers in relational database model. Journal of the ACM, 27(4):664-674, ACM Computer Press, October 1980. 
Mannila H., Toivonen H. and Verkamo A. I. Efficient algorithms for discovering association rules. Proceedings of the AAAI workshop on Knowledge Discovery in Databases, pp 181-192, July 1994.

Mannila H. and Toivonen H. Multiple uses of frequent sets and condensed representations. Proceedings of the KDD conference, pp 189-194, August 1996.

Mannila H. and Toivonen H. Levelwise search and borders of theories in knowledge discovery. Data Mining and Knowledge Discovery, 1(3):241-258, U. Fayyad editor, Kluwer Academic Publishers, September 1997.

Meo R., Psaila G. and Ceri S. An Extension to SQL for mining association rules. Data Mining and Knowledge Discovery, 2(2):195-224, U. Fayyad editor, Kluwer Academic Publishers, June 1998.

Morimoto Y., Fukuda T., Matsuzawa H., Tokuyama T. and Yoda K. Algorithms for mining association rules for binary segmentations of huge categorical databases. Proceedings of the VLDB conference, pp 380-391, August 1998.

Ng R. T., Lakshmanan V. S., Han J. and Pang A. Exploratory mining and pruning optimizations of constrained association rules. Proceedings of the SIGMOD conference, pp 13-24, June 1998.

Pasquier N., Bastide Y., Taouil R. and Lakhal L. Pruning closed itemset lattices for association rules. Proceedings of the BDA conference, pp 177-196, Octobre 1998.

Pasquier N., Bastide Y., Taouil R. and Lakhal L. Discovering frequent closed itemsets for association rules. Proceedings of the ICDT conference, LNCS 1540, pp 398-416, January 1999.

Pasquier N., Bastide Y., Taouil R. and Lakhal L. Efficient mining of association rules using closed itemset lattices. Information Systems, 24(1):25-46, M. Jarke, D. Shasha editors, Elsevier Science, March 1999.

Pasquier N., Bastide Y., Taouil R. and Lakhal L. Closed set based discovery of small covers for association rules. Proceedings of the BDA conference, pp 361-381, Octobre 1999.

Pei J., Han J. and Mao R. Closet: An efficient algorithm for mining frequent closed itemsets. Proceedings of the DMKD Workshop on Research Issues in Data Mining and Knowledge Discovery, pp 21-30, May 2000.

Piatetsky-Shapiro G. and Matheus C. J. The interestingness of deviations. Proceedings of the AAAI workshop on Knowledge Discovery in Databases, pp 25-36, July 1994.

Silberschatz A. and Tuzhilin A. What makes patterns interesting in knowledge discovery systems. Transactions on Knowledge and Data Engineering, 8(6):970-974, IEEE Computer Science, December 1996.

Silverstein C., Brin S. and Motwani R. Beyond market baskets: Generalizing association rules to dependence rules. Data Mining and Knowledge Discovery, 2(1):39-68, U. Fayyad editor, Kluwer Academic Publishers, January 1998.

Srikant R. and Agrawal R. Mining generalized association rules. Proceedings of the VLDB conference, pp 407-419, September 1995.

Srikant R. and Agrawal R. Mining quantitative association rules in large relational tables. Proceedings of the SIGMOD conference, pp 1-12, June 1996.

Srikant R., Vu Q. and Agrawal R. Mining association rules with item constraints. Proceedings of the KDD conference, pp 67-73, August 1997.

Taouil R., Pasquier N., Bastide Y. and Lakhal L. Mining bases for association rules using closed sets. Proceedings of the ICDE conference, p 307, Febuary 2000.

Toivonen H., Klemettinen M., Ronkainen P., Hätönen K. and Mannila H.. Pruning and grouping discovered association rules. Proceedings of the ECML Workshop, pp 47-52, April 1995.

Zaki M. J., Parthasarathy S., Ogihara M. and Li W. New algorithms for fast discovery of association rules. Proceedings of the KDD conference, pp 283-286, August 1997.

Zaki M. J. and Ogihara M. Theoretical foundations of association rules. Proceedings of the DMKD Workshop on Research Issues in Data Mining and Knowledge Discovery, pp 7:1-7:8, June 1998.

Zaki M. J. and Hsiao C.-J. CHARM: An efficient algorithm for closed association rule mining. Technical Report 99-10, October 1999.

Zaki M. J. Generating non-redundant association rules. Proceedings of the KDD conference, pp 34-43, August 2000. 